\title{
ALCANCES DE LA POLÍTICA MONETARIA: MARCO TEÓRICO Y REGULARIDADES EMPÍRICAS EN LA EXPERIENCIA MEXICANA
}

Agustín Carstens y Alejandro Reynoso

Documento de Investigación No. 9705

Dirección General de Investigación Económica BANCO DE MÉXICO 


\title{
Alcances de la política monetaria: \\ marco teórico y regularidades empíricas \\ en la experiencia mexicana
}

\author{
Agustín Carstens y Alejandro Reynoso
}

(El Dr. Agustín Carstens es Director General de Investigación Económica del Banco de México y el Dr. Alejandro Reynoso es Director Asesor de la Junta de Gobierno del Banco de México. Las opiniones contenidas en el presente documento reflejan los puntos de vista de los autores y no necesariamente coinciden con los de la institución en la que laboran.

Los autores agradecen el apoyo y comentarios de Alejandro Gaytán, Alejandro Gonzalez, Alexis Milo, Alejandro Pérez López, Eduardo Rocha, Oscar Sánchez, Porfirio Sánchez y Juan Pedro Treviñ o y reconocen que cualquier error u omisión es responsabilidad exclusiva de quienes firman este documento.)

\begin{abstract}
Sumario
Un tema que ocupa un lugar destacado en el debate sobre la política económica en México es el del papel que debe desempeñ ar la autoridad monetaria para promover la producción, el empleo y la competitividad internacional de la economía en un ambiente de estabilidad de precios. Apoyados en la vasta investigación disponible sobre los alcances de la política monetaria, se desarrolla un modelo muy sencillo que sugiere que, dentro de ciertos rangos, es deseable una política monetaria que acomode las necesidades de liquidez inherentes a las transacciones normales de una economía, y a las necesidades de transferencias intertemporales de recursos de los ahorradores. Sin embargo, rebasados ciertos límites, la expansión monetaria es, en el mejor de los casos, neutral repercutiendo predominantemente en el nivel de precios. Remitiéndose a los informes públicos del Banco de México en los últimos añ os, se sugiere que el criterio de operación adoptado por dicho instituto emisor se aproxima a lo deseable desde el punto de vista del bienestar social. El documento concluye mostrando las relaciones de largo plazo entre agregados monetarios y los principales indicadores del sector real de la economía mexicana, las cuales tienden a confirmar la referida hipótesis de neutralidad y la concomitante recomendación de que el Banco de México persevere en su propósito de abastecer de liquidez indispensable a la economía procurando a la vez la estabilidad de precios.
\end{abstract}


1. Introdicción

2. El modelo

1. La política monetaria en una economía cerrada

1. Las familias

2. Las empresas

3. El sector financiero (tecnología de almacenamiento)

4. El equilibrio general

5. Los alcances de la política monetaria

2. La política monetaria en una economía abierta

1. Las familias

2. Las empresas

3. La balanza de pagos

4. El equilibrio general

5. Los alcances de la política monetaria en la economía abierta

3. Los alcances de la política monetaria en México: hechos estilizados

\section{Conclusiones}

Apéndice 1. Rutina para la determinación de la cantidad óptima de dinero

Apéndice 2. Rutina para la determinación de la cantidad óptima de dinero ante la presencia del señoriaje

Apéndice 3. Determinación de la cantidad de dinero óptima en economía abierta

Referencias 


\section{Introducción}

La política monetaria, sin duda alguna, representa uno de los instrumentos más importantes de que disponen las autoridades para alcanzar el objetivo fundamental de elevar el bienestar de la sociedad. En efecto, las decisiones en materia crediticia, junto con la política fiscal, industrial, comercial, educativa, laboral y salarial hacen, teóricamente, posible diseñ ar programas que ayuden a los agentes económicos a combinar sus capacidades eficientemente para atender sus necesidades de manera oportuna y justa.

Estando en gran medida de acuerdo con el planteamiento normativo propuesto hace más de 40 añ os por Tinbergen [1952], la variedad de opciones de política a la mano de los gobiernos representa un reto y una oportunidad al mismo tiempo. Por un lado, los responsables de las decisiones tienen enfrente de sí la difícil tarea de determinar para qué sirve y para qué no sirve un instrumento dado. Si su juicio es el acertado, estarán utilizando la herramienta más eficaz para resolver un cierto problema, con el concomitante ahorro de recursos y la seguridad de que las metas que se propongan se alcanzarán. Por el contrario, aplicar herramientas que sirven para algo que no esté relacionado con el fin específico que se persiga, terminará costando mucho a la sociedad, al tiempo de que las metas concretas no se lograrán. Este principio, conocido como el de la "clasificación del mercado más eficaz", es la motivación primordial del presente artículo, en el que tratamos de precisar qué tanto se puede hacer desde un banco central y a partir de qué punto la expansión monetaria en vez de ayudar podría convertirse en un obstáculo para el desarrollo nacional.

Para hablar sobre los alcances de la política monetaria es necesario detenerse por un momento a pensar sobre el uso mismo del dinero. Son innegables las contribuciones de autores tales como David Hume [1970] quien propuso por primera vez hace casi 250 añ os el principio de neutralidad de la política monetaria, según la cual los cambios en la cantidad de dinero tienen una limitada capacidad para influir sobre las decisiones de asignación de recursos de los agentes económicos, de tal suerte que dichos cambios acaban por lo regular traduciéndose en variaciones en el nivel general de precios.

La idea de Hume se concentra principalmente en el carácter del dinero como medio de pago, y de sus opiniones se desprende que el poder de las autoridades monetarias para influir persistentemente sobre el bienestar de la sociedad depende de la forma en que el dinero reduzca los costos de transacción.

Samuelson [1958], en el que probablemente sea uno de los artículos más importantes de teoría monetaria que se hayan escrito, llama la atención sobre el carácter de los sistemas financieros como vehículos para permitir a las personas la realización de transferencias intertemporales. Utilizando una metodología de generaciones traslapadas, demuestra que la existencia de dinero puede contribuir a elevar significativamente el nivel de bienestar social. Sin embargo, Samuelson es sumamente cuidadoso al distinguir entre el surgimiento del dinero y la subsecuente expansión del medio circulante. Concretamente, el dinero en sentido estricto, o el sistema financiero visto desde una perspectiva más amplia, son instituciones a través de las cuales las personas pueden ahorrar, lo cual al facilitar las transferencias intertemporales constituye una mejora respecto de situaciones en las cuales tales transferencias fueran imposibles. En este sentido, se afirma que la existencia de dinero es mejor que la falta del mismo, lo cual no significa que una vez que éste exista, más dinero sea mejor. Es aquí donde se regresa a la noción de neutralidad según la cual, cambios en la cantidad nominal de dinero se traducen en incrementos en el nivel de precios, ello sin mayor beneficio para la sociedad.

Contemporáneamente a las ideas de Samuelson, Phillips [1958] elaboró un artículo proponiendo que aunque teóricamente el dinero fuera neutral, en la práctica los 
responsables de la política económica podían utilizar herramientas del lado de la demanda agregada para inducir incrementos en el nivel de actividad y empleo. Específicamente, Phillips presentó evidencia que condujo a pensar que existió una relación inversa relativamente estable entre el nivel de desempleo y la tasa de crecimiento de los salarios en el Reino Unido durante el período 1861-1957. Investigaciones posteriores encontraron una relación similar entre la tasa de desempleo y la de inflación, por lo que esta relación terminó por denominarse la curva de Phillips.

La respuesta a este artículo fue muy entusiasta por parte de los responsables de la política económica en el mundo industrializado; sin embargo, la experiencia pronto sugirió que la capacidad de la política monetaria y fiscal para reducir permanentemente el desempleo era menor que lo inicialmente previsto. Poco pasó para que tal situación fuese reconocida en la literatura, destacando particularmente los artículos de Friedman [1968] y Phelps [1968]. Ambos autores plantearon que cada economía tenía un nivel "natural" de desempleo, y por extensión un nivel "natural" para las demás variables reales. Estos niveles correspondían a sus respectivas tendencias de largo plazo, las cuales estaban determinadas por condiciones estructurales como pueden ser la tecnología, los costos de transacción, eventuales rigideces, fricciones en los mercados y demás arreglos institucionales. Así pues, y en virtud de que los determinantes "fundamentales" de las variables reales se ubicaban del "lado de la oferta", los autores concluyeron que las variables nominales de política económica del lado de la demanda agregada serían incapaces para inducir cambios permanentes en las tasas de crecimiento de la producción o en el nivel de empleo. Por consiguiente, la politica monetaria sólo podría promover transitoriamente la actividad económica, regresando en el largo plazo a la neutralidad ya planteada por Hume tiempo atrás.

Durante los añ os setenta, los investigadores y los funcionarios públicos parecieron caminar por sendas distintas. Los primeros, partiendo de la convicción de que la política contracíclica tenía alcances muy limitados, trataron de entender las posibles causas de una curva de Phillips en el corto plazo, y con ello, establecer con más precisión los límites, en magnitud y en tiempo, dentro de los cuales el activismo monetario y fiscal pudiera justificarse. Por su parte, los responsables de la política económica continuaron instrumentando sus acciones como si la crítica a la multicitada curva de Phillips nunca se hubiera presentado. No será sino hasta después de la experiencia de "estanflación" de aquellos añ os, que se inició un proceso de reconciliación entre ambos grupos de economistas.

Del lado de la teoría, se plantearon numerosas explicaciones para la "ho-neutralidad" transitoria de la política de demanda agregada. De entre todas, acaso la más destacada sea la propuesta por Lucas $[1972,1996]$. El autor señ aló que partiendo de los fundamentos de la economía neoclásica, debería esperarse que individuos racionales asignaran sus recursos de manera distinta a la que, correspondería al equilibrio con perfecta información sólo si éstos carecían de información completa y precisa sobre las decisiones de las autoridades. Es así que si el público anticipa perfectamente cambios en nivel de dinero en circulación, la política monetaria sería neutral, traduciéndose tales variaciones únicamente en cambios en el nivel general de precios.

A las explicaciones de Lucas pueden sumarse otras, como la que sugiere que la existencia de contratos salariales traslapados en el tiempo y sin cláusulas de indización inmediata a cambios en los precios (Fischer [1977], Blanchard [1986] y Taylor [1979]) permite cambios transitorios en precios relativos durante el tiempo en que tarden los trabajadores y empresas en ajustarse a los nuevos niveles de demanda agregada, todo ello con el consiguiente impacto en la asignación de recursos. Otras formas de inflexibilidad de ciertos precios, y de potencial incidencia de la política monetaria en precios relativos es la que se deriva de costos de reetiquetaje, o de renegociaciones salariales intermitentes. (Caplin y Spulber [1987] 
y Caplin y Leahy [1991]).

La brecha que existía entre las opiniones de los investigadores y el punto de vista de los funcionarios a cargo de la política monetaria y fiscal durante los añ os setenta provenía, en buena medida, del hecho de que por un lado Phillips había presentado evidencia en favor de la capacidad de la política activista para reducir eficazmente el desempleo, mientras que por contra sólo se tenían una serie de argumentos relativamente abstractos que no se apoyaban en evidencia suficientemente contundente como para descalificar a la primera (Taylor [1996]). Es por eso que a los documentos seminales de Friedman, Phelps y Lucas siguió un intenso trabajo econométrico.

De acuerdo con Lucas [1981] "Friedman y Phelps no tenían mejor manera que cualquiera de nosotros de pronosticar la inflación de los setenta, sin embargo el punto central de su razonamiento implicaba que una década de inflación alta no debía estar acompañ ada de niveles de desempleo menores que una década de inflación baja. La década de los setenta proporcionó la prueba empírica más exacta que la profesión quizá obtenga nunca, y Friedman y Phelps tuvieron razón".

Fue con Barro [1978], que se tuvo el primer trabajo econométrico que dio sustento a las aproximaciones teóricas de la época. Al descomponer las series observadas de oferta monetaria en los Estados Unidos en dos elementos, uno de tendencia y otro que se identifica con la parte "no anticipada" de la política monetaria, encuentra evidencia de que es tan sólo el segundo el que tenía algún impacto sobre el nivel del producto.

A las pruebas econométricas han seguido estudios menos sofisticados pero igualmente contundentes en sus conclusiones. Por ejemplo, Stiglitz [1997] llama la atención sobre el simple hecho de que entre 1960 y 1980, la tasa de inflación en los Estados Unidos aumentó en 26 de los 32 trimestres en los que la tasa de desempleo estuvo por debajo del 5\%. Asímismo, la tasa de inflación cayó en 24 de los 27 trimestres en los que la tasa de desempleo estuvo por arriba del $7 \%$.

La síntesis de hipótesis aceleracionistas que conducían a predecir la neutralidad de la política de demanda agregada y las evidencias, como la recién comentada, desembocaron en los añ os ochenta en la definición de un indicador que trataría de reflejar la idea inicialmente planteada por Friedman y Phelps del nivel natural de desempleo. Es así como se acuñ a el concepto de la NAIRU (Non Accelerating Inflation Rate of Unemployment) que es la tasa de desempleo consistente con una inflación estable.

Poco a poco la idea de la NAIRU fue saliendo de las universidades para aparecer como una referencia común en los reportes de bancos centrales, empresas de análisis y corredurías, lo cual contribuyó a construir un consenso, más o menos general, acerca de la neutralidad de la política económica en el largo plazo (Gordon [1996]).

De hecho, la NAIRU se ha convertido en una herramienta muy útil para muchos analistas cuando se trata de emitir un juicio sobre el eventual sobrecalentamiento de la economía. Así, es común ver que los mercados financieros esperan ávidamente las cifras de desempleo en los Estados Unidos, por ejemplo, para compararlas con la NAIRU y con ello diagnosticar la presencia o no de presiones inflacionarias.

La popularización de la NAIRU como instrumento de trabajo en el sector financiero de las naciones industrializadas ha tenido una retroalimentación muy positiva en el ámbito académico, toda vez que los trabajos econométricos sobre el tema se han multiplicado. Esto último a su vez ha facilitado la convergencia entre las predicciones de la teoría y la persuasión de quienes hacen política económica.

En los añ os noventa, las discusiones en las naciones desarrolladas cuentan ya con un 
punto de partida muy distinto al de la década precedente. Habiéndose aceptado la hipótesis aceleracionista, según la cual el nivel de desempleo puede caer por debajo de su nivel natural sólo si la inflación crece a tasas crecientes, la atención ahora se centra en medir la relación entre la tasa de aceleración de los precios y la tasa de desempleo, así como en determinar con mayor precisión como es que la NAIRU ha cambiado a través del tiempo.

En relación con el primer aspecto, es generalmente aceptado que si se corre una regresión del cambio en la tasa de inflación contra valores rezagados de la tasa de desempleo, independientemente de otras variables incluidas para mejorar el ajuste, los estadísticos "t" asociados a la tasa de desempleo son regularmente significativos para niveles de significacia del 1\%. (Gordon [1997] y Staiger, Stock y Watson [1997]).

Por su parte, no sólo los analistas sino las propias autoridades monetarias toman sus decisiones en la inteligencia de que la NAIRU puede estar cambiando. Algunos ejemplos recientes de estas actualizaciones pueden verse en Gordon [1997], King, Stock y Watson [1995], y Staiger, Stock y Watson [1996, 1997]).

Desafortunadamente para países como el nuestro, el interés académico en las condiciones que podrían conducir a la no-neturalidad de la política monetaria en el corto plazo se concentró por mucho tiempo casi exclusivamente en la experiencia de las economías industrializadas.

No obstante lo anterior, lo ocurrido durante los añ os de alta inflación en los setenta y los ochenta fue lo suficientemente serio como para atraer la atención de algunos académicos aquí y en universidades de Estados Unidos y Europa, lo cual permitió hace poco más de una década, empezar a hacer reflexiones más serias sobre las consecuencias de la política monetaria. De esta forma, la investigación sobre las experiencias monetarias en nuestra región avanzó en dos direcciones muy interesantes.

En primer lugar, fue posible demostrar que los países que utilizaban la política de demanda agregada para provocar un mayor crecimiento de la actividad y el empleo, partiendo de niveles de inflación de dos dígitos, muy pronto transitaban a la hiperinflación sin que en el trayecto hubiesen alcanzado los beneficios previstos. Por ejemplo, Ball, Mankiw y Romer [1988] probaron que la curva de Phillips de corto plazo era menos elástica en países con inflación alta.

En segundo lugar, la experiencia latinoamericana permitió incorporar al debate sobre el papel de la política monetaria al sector externo; particularmente en lo relativo al mecanismo de transmisión que existe entre la política monetaria, el tipo de cambio y el nivel de precios. Específicamente, aún cuando la pregunta de si la autoridad monetaria sería capaz de influir de manera permanente sobre los precios relativos de la economía es en el fondo la misma que planteaba la investigación acerca de la existencia o no de una curva de Phillips, el énfasis en economías abiertas y pequeñ as se enfocó en la capacidad que las autoridades tuvieran para influir persistentemente sobre un precio relativo en particular: el tipo de cambio real. (Bacha [1979] y Dornbusch [1982]).

El debate sobre este tema produjo dos paradigmas en materia de política económica claramente opuestos. Por un lado, quienes planteaban que la estabilidad macreoconómica requería necesariamente de anclas nominales bien definidas y visibles, como sería el tipo de cambio nominal y/o alguna clase de agregado monetario; y por el otro lado, quienes afirmaban que el crecimiento económico sostenible en economías abiertas dependía crucialmente de contar con un tipo de cambio competitivo, de forma que tal variable se ajustara de acuerdo con los diferenciales de inflación interna e internacional. Parafraseando lo anterior, diríamos que una posición es de quienes consideran que no es posible utilizar una variable nominal, como el tipo de cambio nominal o la cantidad de dinero, para influir 
persistentemente sobre variables reales, mientras que otros opinan que no sólo es posible sino deseable utilizar el tipo de cambio nominal para alcanzar objetivos de crecimiento y empleo.

En contraste con el consenso que existe en los países más avanzados, es claro que en América Latina el debate aún se antoja lejos de su conclusión, por lo que la revisión cuidadosa de las experiencias y el desarrollo del marco teórico para economías abiertas son fundamentales para establecer, con base científica, si los principios que se consideran válidos en la literatura para otros países pueden extenderse a nuestra región.

Por fortuna, el trabajo disponible arroja ya cierta claridad sobre la respuesta. Por ejemplo, Blejer y Fernández [1980] establecen que en el caso de México un aumento no anticipado en el crecimiento del crédito doméstico provoca una expansión en el sector de no-comerciables y una caída en la producción de bienes comerciables. Estos autores concluyen que este efecto es el equivalente al de la curva de Phillips de corto plazo en una economía cerrada, y que el mismo se desvanece una vez que las expectativas se ajustan.

Por otro lado Reynoso [1989], muestra que a pesar de que la presencia de las rigideces propias de economías en desarrollo excesivamente reguladas, como el control de precios, de cambios o de tasas de interés, la experiencia demuestra que el activismo de la política monetaria y fiscal tiene efectos limitados sobre el nivel de actividad y empleo en virtud de las propias restricciones presupuestales del sector público, así como de la acotada capacidad del sector externo para financiar los desequilibrios que derivan de la intervención del gobierno en los mercados.

Aspe [1993], haciendo un recuento de la experiencia mexicana de inflación y estabilización, llama la atención sobre la forma en que se acelera la inflación en períodos en los que se establecieron reglas de desliz cambiario que pretendían subvaluar al tipo de cambio en términos reales. Menciona que además de las consecuencias que derivaban de la transmisión directa del tipo de cambio a los precios de los bienes comerciables, el hecho mismo de tener una inflación mayor condujo a la contracción de la duración de los contratos salariales y al aumento de la inercia inflacionaria. Así, las consecuencias estructurales de una meta de tipo de cambio real eran la indización de la economía y la eventual tendencia hiperinflacionaria.

Carstens y Gil Díaz [1996] presentan un modelo que reproduce las fórmulas de indización de precios públicos y privados en México para mostrar que el pass-through del tipo de cambio a precios, una vez que se combina con la restricción presupuestal del gobierno para reflejar los costos de los consiguientes subsidios, es lo suficientemente elevado como para reconocer las bondades de las anclas nominales en la estabilización macreoconómica. Sus conclusiones, pueden extenderse con el ejercicio inverso para decir que una política de devaluaciones que tuviera por objetivo alcanzar un cierto nivel de tipo de cambio real terminaría por trasladarse a la inflación con un impacto breve y sólo transitorio sobre el tipo de cambio real.

El caso de México relatado en las referencias anteriores tiende a repetirse en otros países, especialmente en aquéllos que parten de niveles de inflación en el rango de dos dígitos, según describen Kamin [1996] y McDonald [1997]. Inclusive Calvo, Reinhart y Végh [1995], sugieren que experiencias con objetivos de tipo de cambio real conducen generalmente a inflaciones persistentes, altas y volátiles. Más aún ellos llegan al punto de establecer que experiencias basadas en un tipo de cambio real objetivo que generalmente se consideran exitosas, como la chilena, tarde o temprano acaban por abandonarse ante situaciones tales como la fuerte entrada de capitales de principios de los añ os noventa.

En el terreno de la discusión teórica, Adams y Gros [1986] proponen un modelo monetario de determinación del nivel de precios para analizar las consecuencias, 
sobre la tasa de inflación, de adoptar una regla de tipo de cambio real constante (conocidas como reglas PPP). Reconocen que aunque una regla de deslizamiento cambiario pudiera mejorar la balanza de pagos en el corto plazo, llevaría a la perdida del control de la inflación. La razón radica en que la regla implica indizar tanto el tipo de cambio nominal como la oferta monetaria al nivel de precios, provocando que no exista un ancla nominal que modere la inflación en el largo plazo. Este modelo establece además que la incorporación de topes al crédito primario, en combinación con reglas de PPP, resulta insuficiente para construir un ancla nominal eficaz.

Montiel y Ostry [1991] enfatizan sobre los efectos en la inflación y la cuenta corriente derivados de adoptar una regla de PPP. En su modelo, el crecimiento de los precios afecta el patrón de consumo y la cuenta corriente por la vía del impuesto inflacionario. Sugieren que la tasa de inflación de largo plazo está determinada por la condición de que el impuesto inflacionario genere un nivel de demanda consistente con el equilibrio en el mercado de no-comerciables y la regla de PPP. Por lo tanto, para lograr un nivel objetivo de un tipo de cambio real más depreciado es necesario elevar la recaudación por impuesto inflacionario lo cual conduce a más inflación si la demanda por dinero es inelástica con respecto a la tasa de interés.

En un documento posterior, Montiel y Ostry [1992] introducen el control de cambios como un posible instrumento para conciliar una regla de PPP con la estabilidad de precios. En su propuesta, el control de cambios toma la forma de un tipo de cambio dual con un tipo de cambio oficial sujeto a una regla y otro flotante. Mientras el control de cambios prevenga la interacción entre estos dos mercados, la política monetaria puede funcionar nuevamente como ancla nominal. Sin embargo, también su modelo predice que dada una regla de PPP, la brecha entre el tipo de cambio oficial y el flotante aumenta continuamente lo que hace eventualmente imposible controlar los derrames de un mercado a otro.

Por su parte, Lizondo [1993] explora los posibles méritos de instrumentos que segmenten los mercados financieros doméstico y externo mediante un modelo que contempla su imperfecta sustituibilidad. Sin embargo, al igual que en el caso anterior se muestra que inevitablemente una regla de PPP termina por traducirse en mayor inflación en la medida en que exista cierta conexión, por pequeñ a que sea, entre ambos mercados.

Poniendo en perspectiva lo que hemos dicho hasta aquí, debe quedar claro que la agenda de investigación sobre la neutralidad de la política monetaria y cambiaria en economías en vías de desarrollo, debe transitar con rapidez de las propuestas teóricas hacia la acumulación y divulgación de la evidencia empírica, y de las universidades a las instituciones de análisis que forman opinión pública, ello con el propósito de evitar discusiones estériles que minen la capacidad de las autoridades para aplicar las herramientas idóneas en la promoción del crecimiento con estabilidad.

Teniendo esto en mente, el resto del presente artículo busca contribuir al debate desde el punto de vista de la economía mexicana. A tal fin se incluyen otras dos secciones. La primera de ellas contiene un modelo en el cual se resaltan los efectos y las limitaciones de la política monetaria cuando hay mercados financieros incompletos, tanto en una economía cerrada como en una economía abierta.

La última sección, por su parte, enfoca el tema de los alcances de la política monetaria desde el punto de vista de la experiencia mexicana de los últimos añ os. 


\section{El modelo}

En esta sección se presenta un modelo neoclásico muy sencillo que se apoya en las ideas de Samuelson [1958] para resaltar los alcances de la política monetaria. Aquí nos interesa destacar el hecho de que el dinero, interpretado en un sentido amplio como el conjunto de instrumentos que permiten a las personas realizar transacciones corrientes y transferencias intertemporales, presta un servicio que contribuye al bienestar social.

Aunque el modelo conserva los supuestos fundamentales que normalmente se refieren a economías ideales, como son la racionalidad de los agentes económicos y perfecta información, incluye el supuesto de que hay un mercado incompleto: el de fondos prestables.

El fenómeno que se intenta reproducir es lo que ocurre cuando una economía, que no cuenta con los vehículos adecuados para hacer transferencias intertemporales, desarrolla su sector financiero, visto en este caso como la expansión de los agregados monetarios, en términos reales.

A lo largo de este ejercicio, estará implícita la noción de que el desarrollo del sistema financiero no es un fenómeno discreto, sino que va dándose gradualmente. En consecuencia, por un tiempo los agentes actuarán como si sus posibilidades de transferir recursos a través del tiempo estuviesen racionadas.

De aquí se desprenderá que en economías con mercados incompletos la política monetaria no tiene por que ser neutral, en la medida en que ella acomode necesidades reales de inversión y ahorro del público. Sin embargo, también el modelo mostrará que una vez que tales necesidades sean satisfechas, una política encaminada a la expansión de los agregados monetarios terminará por repercutir exclusivamente sobre el nivel general de precios.

Se presenta primero el ejercicio para una economía cerrada y luego se extiende para una economía pequeñ a y abierta con el propósito de puntualizar los alcances de la política monetaria sobre la producción, las tasas reales de interés, tipo de cambio real y bienestar social.

\section{La política monetaria en una economía cerrada}

1. Las familias

La economía está compuesta por consumidores con una función de utilidad tipo Cobb-Douglass, cuyo bienestar deriva del consumo y del ocio en los dos períodos que abarca esta economía.

$$
\begin{gathered}
U=\left(c_{1}^{a}\left(24-a_{1}\right)^{1-a}\right)^{o}\left(c_{2}^{a}\left(24-a_{2}\right)^{1-a}\right)^{(1-p)} \\
0 \leq \alpha \leq 1 ; 0 \leq p \leq 1
\end{gathered}
$$


número de horas trabajadas en el mismo. Para simplificar, establecimos que cada período tiene un máximo de horas trabajables de 24.

Las familias, actuando como tomadoras de precios, llevan a cabo sus decisiones de consumo, ahorro y oferta de trabajo en cada período. Conviene señ alar que las familias perciben ingresos no sólo a través de la nómina sino por concepto de las ganancias que generen las empresas de las cuales ellos son accionistas.

Para efectuar su ahorro, suponemos como punto de partida que los individuos no tienen restricción para acumular dinero por el cual, además, no reciben intereses. Sin perjuicio de lo anterior, el modelo puede modificarse con facilidad para interpretar al dinero como instrumento de ahorro en un sentido amplio. Consiguientemente, la restricción presupuestal puede expresarse como:

$$
p_{1} c_{1}+p_{2} c_{2}+r-p_{1} s_{1} c_{1}-p_{2} s_{2} c_{2}-p_{1} s_{1} \cdot s_{1}-p_{2} s_{2} \cdot s_{2}=0 \text {, }
$$

con $\mathrm{p}_{\mathrm{t}}$ correspondiendo al nivel de precios en cada período; $\mathrm{s}_{\mathrm{t}}$, los salarios reales y $\pi_{\text {, }}$ las ganancias de las empresas expresadas en términos reales. La variable $\tau$ se refiere a los impuestos que el gobierno podría cobrar a las familias.

2. Las empresas

El sector productor está también compuesto por pequeñ as empresas tomadoras de precios, cuya tecnología para cada período está dada por

$$
y_{1}=l_{1}^{\beta}, y_{2}=l_{2}^{\beta}, 0 \leq \beta \leq 1 .
$$

Las empresas no acumulan inventarios y utilizan solamente al factor trabajo en la producción, donde l es la cantidad empleada del mismo.

Conviene adelantar aquí que el efecto que se lograría si las empresas acumularan inventarios o utilizaran parte de la producción pasada como insumo, se obtiene incorporando al gobierno como "agente almacenador", según veremos. Por ahora, baste señ alar que el sector productor busca maximizar sus ganancias, definidas para cada período como: 


$$
s_{t}=p_{t} y_{t}-p_{t} s_{t} l_{t}
$$

3. El sector financiero (tecnología de almacenamiento)

Antes de entrar a los detalles del equilibrio general, vale la pena reflexionar un poco más sobre el tipo de transacciones a las que nos referiremos. Tenemos por un lado a las familias que con su ingreso pueden decidir entre comprar bienes o acumular dinero; ese dinero tiene una importancia fundamental para ellos, toda vez que es un mecanismo que les permite transferir parte de sus ingresos presentes hacia consumo futuro. El gobierno, por su parte, a través del banco central emite el dinero. Ahora bien, supondremos que las autoridades adquieren los bienes no con el propósito de consumirlos sino de almacenarlos.

De esta forma, en el período 1, la demanda de bienes estará dada por el consumo privado más los bienes que el gobierno acumulará por "encargo" del sector familias. La oferta de bienes en esta etapa provendrá de las decisiones optimizadoras de las empresas.

En el período 2, las familias recibirán ingresos correspondientes a tal período pero, al momento de decidir su gasto tomarán en cuenta no sólo que tienen los ingresos derivados de su trabajo y de los dividendos que pagan las empresas sino que también contarán con los saldos nominales acumulados en el período anterior para gastarlos en bienes.

El gobierno, por su parte asumirá su obligación que recoger el dinero emitido a cambio de los bienes "almacenados" para el público. Es decir, aquí el gobierno desempeñ a claramente una función que, lejos de interferir, tiene como propósito ampliar las posibilidades de elección de las familias. Como resultado de lo anterior, la demanda agregada en el período 2 estará dada por el consumo privado, mientras que la oferta provendrá de las empresas más los inventarios que a través de la acumulación que hizo el gobierno regresarán al mercado.

En lo tocante a la tecnología a través de la cual los bienes "almacenados" por el gobierno se transforman de un período a otro, trabajaremos con el supuesto simplificador de que un bien en el período 1 se transforma, tras su almacenamiento, en precio1/precio 2 unidades de bien 2. Esto tiene como consecuencia que las utilidades de operación del instituto emisor sean cero, de tal suerte que no sea necesario cobrar impuestos o regresar subsidios a la sociedad, es decir que $\tau=0$.

En el apéndice 2 de este documento se ofrece al lector una rutina para MapleV (Para mayor detalle ver Reynoso [1997].) que le permitirá repetir los ejercicios que aquí presentamos suponiendo que una unidad de bien 1, almacenado en el período 1 , se convirtiera "uno a uno" en bienes del período 2, y notará que en lo esencial los resultados no cambian. Conviene resaltar que en este caso surge el "señ oriaje" como una fuente de ingresos públicos, los cuales regresan al sector familias en forma de subsidios $(\tau<0)$.

A partir de estas extensiones al escenario base, resultará obvio que si bien el modelo no considera explícitamente que la intermediación 
financiera tenga como propósito el financiamiento de la inversión, bastará con reinterpretar a la tecnología de almacenamiento como una tecnología de producción, y ver al gobierno como un productor. Es decir, estamos hablando de una economía en la que el sector financiero juega precisamente un papel de intermediación entre ahorro e inversión.

4. El equilibrio general

Con los elementos que hasta aquí se han enumerado, se procede a obtener las funciones de oferta y demanda agregada de la economía, las ofertas y demandas de trabajo y la condición de equilibrio en el mercado de fondos prestables. Los detalles algebraicos se obvian toda vez que el lector podrá revisar paso a paso este ejercicio aplicando las instrucciones de MapleV que se listan en el apéndice 1; sin embargo, conviene hacer algunas observaciones. Las funciones de exceso de demanda de bienes y factores que resultan tienen la siguiente estructura básica:

\begin{tabular}{|c|c|}
\hline $\begin{array}{l}E D B_{1}=p_{1} c_{1}\left(p_{1}, p_{2}, s_{1}, s_{2}, \pi_{1}\left(p_{1}, s_{1}\right), \pi_{2}\left(p_{2}, s_{2}\right)\right)+M-p_{2} y_{1}\left(p_{1}, s_{1}\right) \\
E D B_{2}=p_{2} c_{2}\left(p_{1}, p_{2}, s_{1}, s_{2}, \pi_{1}\left(p_{1}, s_{1}\right), \pi_{2}\left(p_{2}, s_{2}\right)\right)-p_{2} y_{2}\left(p_{2}, s_{2}\right)-M \\
E D T_{1}=p_{1} s_{1} u\left(p_{1}, p_{2}, s_{1}, s_{2}, \pi_{1}\left(p_{1}, s_{1}\right), \pi_{2}\left(p_{2}, s_{2}\right)\right)-p_{1} s_{1} l_{1}\left(p_{1}, s_{1}\right) \\
E D T_{2}=p_{2} s_{2} u_{2}\left(p_{1}, p_{2}, s_{1}, s_{2}, \pi_{1}\left(p_{1}, s_{1}\right), \pi_{2}\left(p_{2}, s_{2}\right)\right)-p_{2} s_{2} l_{2}\left(p_{2}, s_{2}\right) \\
E D D=p_{2} y_{1}\left(p_{1}, s_{1}\right)-p_{1} c_{1}\left(p_{1}, p_{2}, s_{1}, s_{2}, \pi_{1}\left(p_{1}, s_{1}\right), \pi_{2}\left(p_{2}, s_{2}\right)\right)-M\end{array}$ & (5) \\
\hline
\end{tabular}

$E B_{t}$ es la función del valor del exceso de demanda de bienes en el período $t, E D T_{t}$ es la función del valor del exceso de demanda en el mercado de trabajo en el período $t$, y EDD es el exceso de demanda de dinero. Por inspección, esta última ecuación es igual al valor absoluto del exceso de demanda de bienes en el período 1, lo cual implica que tenemos efectivamente un sistema de 4 ecuaciones para determinar el equilibrio general. Sin embargo, en virtud de la ley de Walras (véase en el apéndice 1, línea 27), la suma de las cuatro ecuaciones que quedan se anula. Baste ver para esto último que tal adición es igual a la restricción presupuestal (2), la cual es igual a cero por construcción.

Lo anterior, tiene implicaciones muy interesantes respecto al impacto de la política monetaria. En efecto, dada la cantidad nominal de dinero, la solución simultánea de tres mercados nos permite determinar los precios relativos $p_{2} / p_{1}$ 
y los salarios reales $s_{1}$ y $s_{2}$. Esto quiere decir, que aún conociendo la cantidad nominal de dinero no es posible establecer un nivel general de precios.

Así pues, si tomamos como numerario al bien producido y consumido en el período $1\left(p_{1}=1\right)$, la política monetaria consistente en establecer una cierta cantidad nominal de dinero en circulación $(M)$, de hecho equivale a establecer un nivel real de dinero. Dado lo anterior, la pregunta que queda por hacer es si para tal nivel "real" de dinero en circulación existe un vector de precios $\left(p_{2} / p_{1}, s_{1}, s_{2}\right)$ de tal manera que los mercados de bienes y factores se equilibren.

Veremos que la respuesta es afirmativa para más de un monto de dinero en circulación. De hecho, para ciertas funciones de utilidad y tecnologías como las que se proponen en el presente documento, encontramos un continuo de saldos nominales (y reales) para los cuales existe un equilibrio. Sin embargo, también encontramos que se llega a un cierto punto a partir del cual, incrementos sucesivos en la cantidad de dinero no son compatibles con un equilibrio.

La razón proviene del fenómeno que advertíamos al inicio de esta sección. Si interpretamos al sector financiero como un "almacén", y la gente encuentra ventajas, ya sea por razones de preferencias, tecnología o ambas, para efectuar transferencias intertemporales, la gente aprovechará la posibilidad de "depositar" sus bienes en tanto dicho "almacén" sea menor que sus deseos de "ahorro". Sin embargo, una vez que el multicitado "almacén" crezca por encima del nivel que la sociedad juzga adecuado, empezará a darse un exceso de capacidad instalada que no podrá corregirse más que "achicándolo". En el caso del dinero, más allá de cierto nivel de circulación, expansiones adicionales tendrán que tener como contrapartida aumentos en el nivel de precios para regresar los saldos reales a su nivel máximo de equilibrio.

Un último aspecto a considerar es que, entre un nivel de saldos igual a cero y el máximo compatible con el equilibrio, incrementos en los saldos reales están aparejados de aumentos en el nivel de bienestar de la sociedad, de tal suerte que la cantidad máxima de dinero compatible con el equilibrio es también la cantidad óptima.

5. Los alcances de la política monetaria

A través de una serie de ejemplos es posible ilustrar con más claridad lo que sucede en esta economía. El lector, utilizando los apéndices que se presentan al final del documento, podrá efectuar ejercicios similares para distintos valores de los parámetros de preferencias y tecnología.

Como referencia supondremos que $\alpha=0.5, \beta=0.5, \theta=0.25$. Con ello lo primero que hacemos es determinar cuál es el nivel máximo de saldos reales para el cual existe un vector de precios relativos que permita el equilibrio general. Puede determinarse que tal monto es 2.75 unidades monetarias, lo cual, en el equilibrio, representa alrededor del $75 \%$ del PIB correspondiente al primer período, como era de esperarse por la forma de la función de utilidad y el valor del parámetro $\theta$. 


\section{Gráfica 2.1}

PIB en el primer período

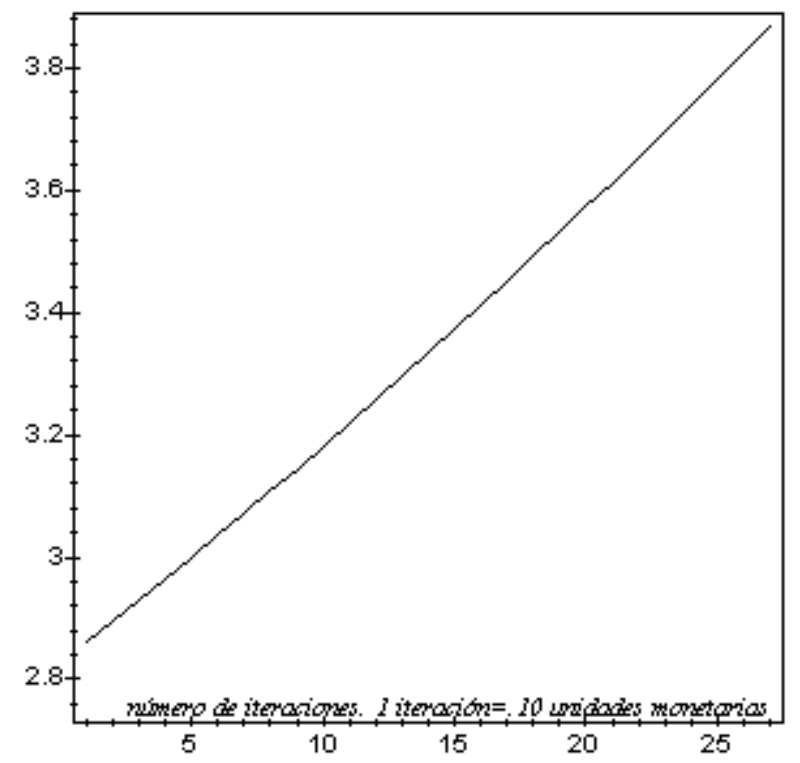

Al experimentar con valores superiores a 2.75 no es posible encontrar un equilibrio, por lo cual incrementos adicionales a la cantidad nominal de dinero en circulación deben compensarse por aumentos proporcionales en los precios de tal suerte que los saldos acumulados, como proporción del producto, no rebasen el $75 \%$. Dado eso, la pregunta es qué sucede antes del máximo nivel de saldos monetarios sostenible por un equilibrio. Como mencionamos previamente, resultará que puede haber más equilibrios los cuales pueden acomodarse según su incidencia en el bienestar social. A tal fin efectuamos varios cálculos para determinar los efectos de la política monetaria en el bienestar, la producción y el consumo intertemporal. 


\section{Gráfica 2.2}

\section{Consumo en el segundo período}

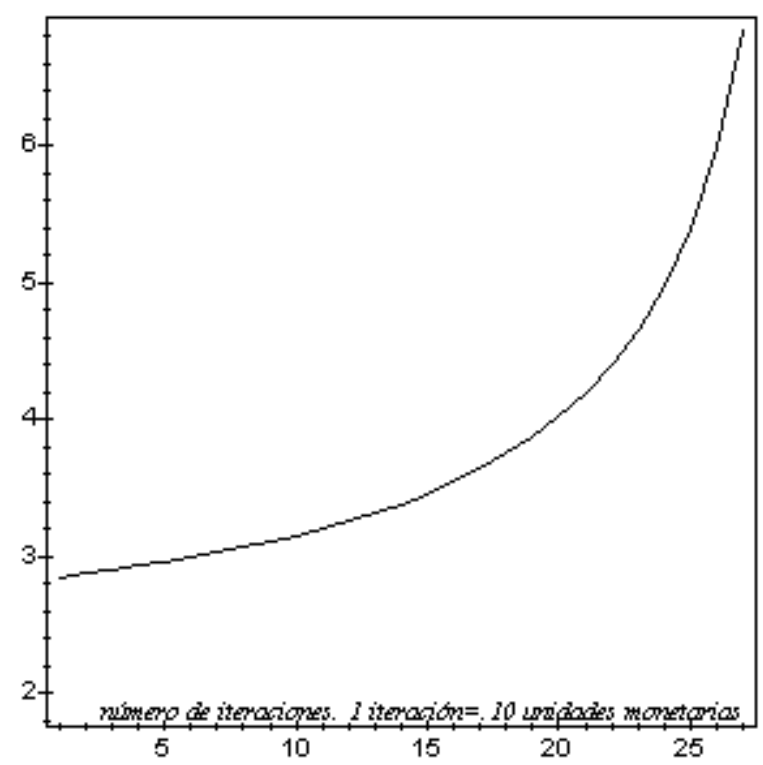

Por un lado, en el rango de equilibrios posibles, los aumentos en la cantidad de dinero están asociados a incrementos en los niveles de producción en el período 1. Ello, principalmente como resultado de que los individuos tratarán de aprovechar las oportunidades de ahorro que antes no tenían en virtud de que sus preferencias son tales que existe la posibilidad de elevar el bienestar mediante la transferencia de ocio presente y consumo presente para convertirlo en consumo futuro. En el equilibrio, la cantidad de producción y empleo se incrementan, si bien a un ritmo menor al crecimiento en la cantidad de dinero.

Por su parte, el consumo en el período 2 se incrementa con rapidez reflejando la combinación de dos efectos, por un lado el aumento en la producción y por otro la sustitución de consumo presente por consumo futuro.

En cuanto al nivel de precios, vemos que antes del nivel óptimo de dinero, el nivel de precios del período 1 no se modifica, sin embargo, los precios relativos en el segundo período disminuyen en gran medida por las fuertes transferencias de producción del período y su correspondiente efecto sobre la oferta agregada. Este comportamiento también puede interpretarse como el rendimiento que el público percibe de su ahorro.

La combinación de todos estos efectos se traduce en una relación directa entre el nivel de bienestar y la cantidad óptima. Interpretando estos resultados podemos regresar al planteamiento que se hacía al principio de esta sección, según el cual, no sólo es entendible sino deseable que las autoridades hagan un esfuerzo por elevar gradualmente la profundización financiera en economías con mercados en desarrollo. En este sentido, la política monetaria adecuada es la que procura abastecer de liquidez a la sociedad en función de sus necesidades de crecimiento. 


\section{Gráfica 2.3}

$(\mathbf{p 1}-\mathbf{p 2}) / \mathbf{p} 2$

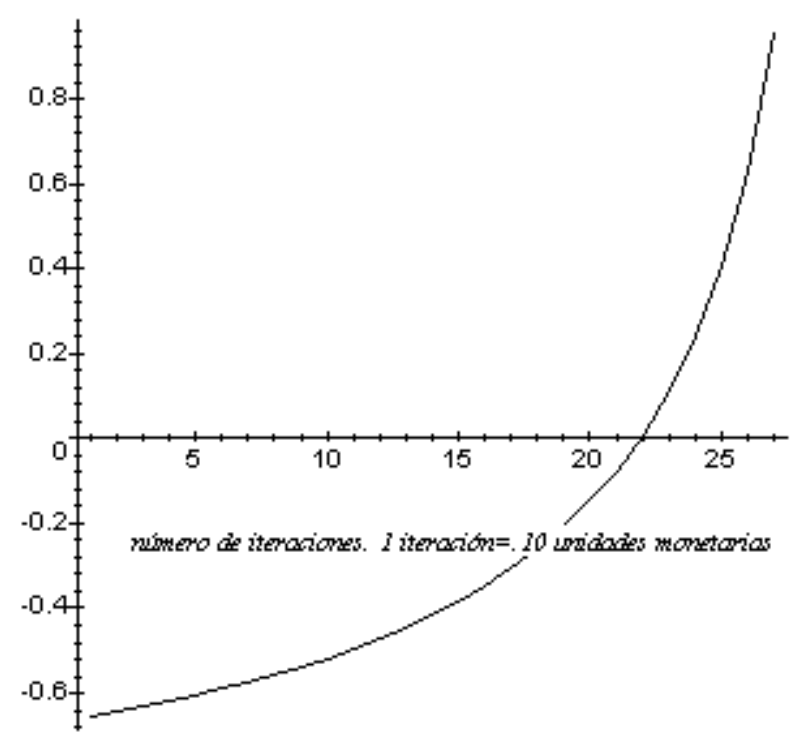

Dicho en otras palabras, la neutralidad del dinero significa que las autoridades financieras no pueden impulsar a la economía por encima del nivel que nosotros asociamos en este modelo como la cantidad óptima; sin embargo, ello no quiere decir que tales autoridades deban mantenerse pasivas e ignorantes de lo que sucede en el sector real. Por el contrario, tienen un papel fundamental en la promoción de los mercados financieros, lo cual debe traducirse tanto en un ordenado crecimiento del dinero de alto poder como en facilitar y vigilar la innovación en los demás ámbitos del sector financiero. 


\section{Gráfica 2.4}

\section{Bienestar Social}

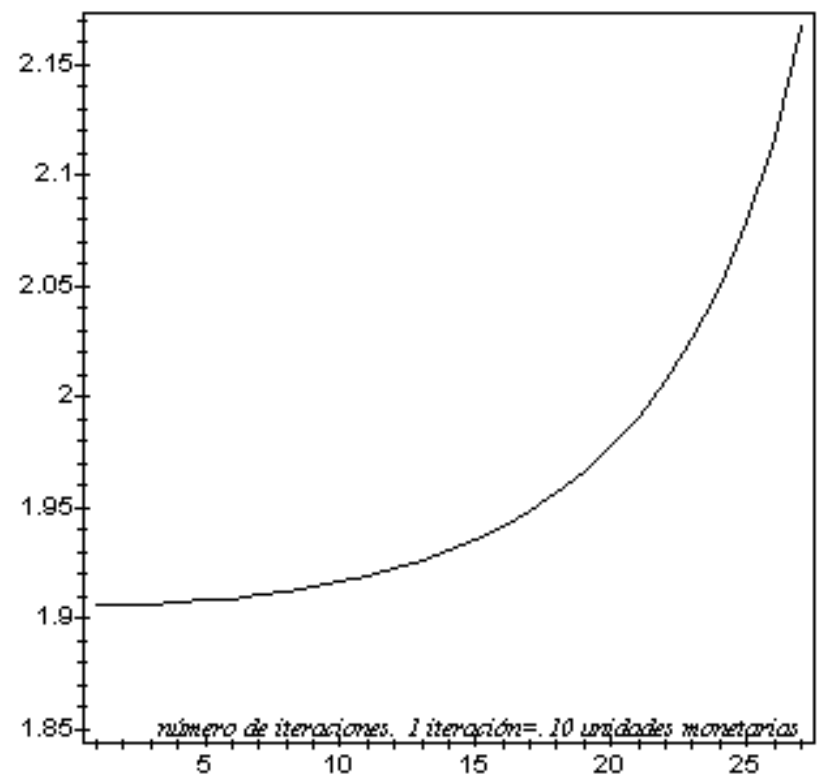

2. La política monetaria en una economía abierta

El modelo presentado en la sección anterior puede generalizarse al caso de una economía abierta mediante la incorporación de un nuevo bien que suponemos sólo se produce en el extranjero. Por su parte, permitimos que los bienes producidos domésticamente sean exportables.

1. Las familias

Las familias consumirán tanto bienes producidos domésticamente c como bienes importados im en cada uno de los dos períodos. Como punto de partida suponemos que pueden hacer todo tipo de transferencias intertemporales tanto en bienes domésticos como bienes importados. De esta forma las expresiones (6) y (7) corresponden a la función de utilidad y la restricción presupuestal.

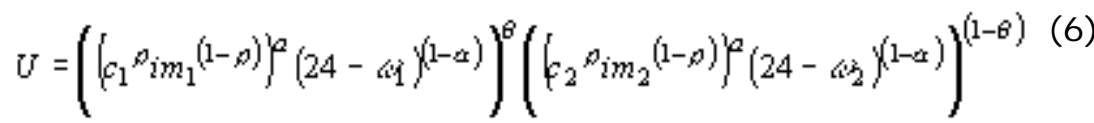

$$
\begin{aligned}
& 0 \leq \theta \leq 1 ; 0 \leq \theta \leq 1 ; 0 \leq \theta \leq 1
\end{aligned}
$$


El precio de los bienes externos está dado por el tipo de cambio $e_{t}$ en cada período. Por su parte, la oferta de los bienes importados es ilimitada siendo la única restricción, como veremos más adelante, el equilibrio en la balanza de pagos.

2. Las empresas

Suponemos que las empresas pueden producir bienes para el mercado interno o bienes para el mercado externo. Las funciones de producción de cada tipo de bienes están dadas por (8). Para simplificar supondremos que las tecnologías son idénticas tanto para los bienes "no comerciables" destinados al consumo de las familias como de los bienes

exportables,

$$
y_{1}=l_{y 1}^{\beta}, y_{2}=l_{y 2}^{\beta}, x_{1}=l_{x 1}^{\beta}, x_{2}=l_{x 2}^{\beta}, 0 \leq \not \beta \leq 1
$$

donde $y_{t}$ se refiere al sector de no comerciables y $x_{t}$ a la producción para exportaciones.

3. La balanza de pagos

Existen distintas formas de modelar la balanza de pagos, según si se considera que es posible tener acceso a capitales del exterior. Nuestro modelo funcionará como si el desarrollo de los mercados financieros internos avanzara al mismo paso que el acceso a los mercados internacionales de capitales. De la misma forma en que se hizo al describir la restricción presupuestal de las familias donde el punto de partida consiste en establecer que los individuos puedan transferir tantos recursos como quieran entre un período y otro, aquí se supone que existe crédito externo suficiente para cubrir el déficit de un período siempre y cuando se pague en el siguiente mediante un superávit.

Conviene mencionar aquí que el tipo de cambio es una variable endógena que se puede ajustar libremente. Así tenemos que la condición de equilibrio en la balanza de pagos es la siguiente:

$$
B D P=e_{1}\left(i m_{1}-x_{1}\right)+e_{2}\left(i m_{2}-x_{2}\right)=0
$$

4. El equilibrio general

Las condiciones del equilibrio que estaremos analizando, obviando por las razones ya expuestas a la condición de equilibrio en el mercado de 
fondos prestables, son las siguientes:

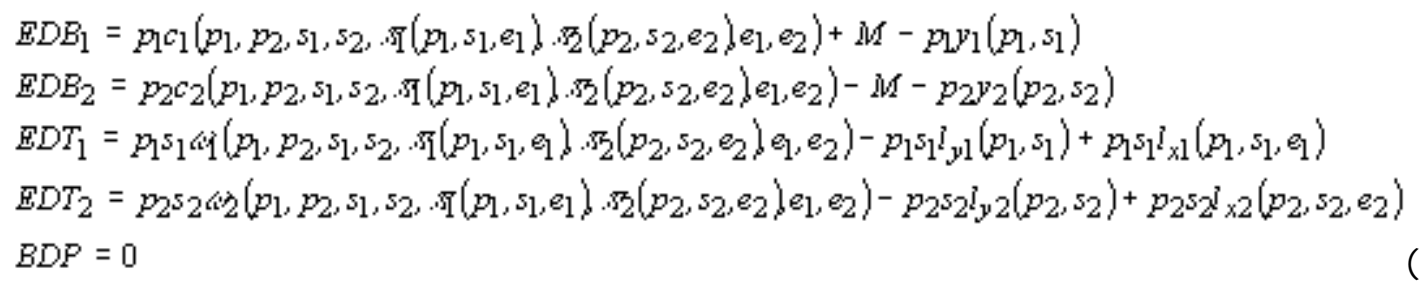

5. Los alcances de la política monetaria en la economía abierta

En el apéndice 3 el lector encontrará las instrucciones de MapleV para una economía abierta las cuales le ofrecen la flexibilidad que desee para experimentar con distintas alternativas, no sólo en lo tocante a los valores de los parámetros de preferencias y tecnología sino en cuanto a la posibilidad de analizar distintas maneras de modelar las restricciones de balanza de pagos.

Tomaremos el caso en el que $\alpha=\rho=\beta=0.5$ y $\theta=0.25$, con el propósito de concentrar nuestra atención en las funciones del dinero descritas en párrafos previos.

El resultado más importante tiene que ver con lo que sucede con el tipo de cambio nominal, y consiguientemente con el tipo de cambio real cuando la cantidad de dinero disponible para hacer transferencias intertemporales se incrementa.

El lector podrá comprobar que el equilibrio que corresponde para los parámetros propuestos tiene como característica que $p_{1}=e_{1}$ y $p_{2}=e_{2}$ para cualquier valor de $M$. Esto quiere decir que el tipo de cambio real es totalmente insensible a la política monetaria.

En la gráfica siguiente se reporta la "tasa de depreciación nominal", la cual es la relación $\left(e_{2}-e_{1}\right) / e_{1}$. En este caso no tenemos por que esperar que la referida cantidad sea fija, en virtud de que los precios relativos se modifican cuando la gente encuentra la posibilidad de hacer transferencias intertemporales en el sector doméstico. La forma de esta gráfica muestra que en la medida en que se expande el sector financiero, los precios de los bienes comerciables y no comerciables, expresados en términos de los precios del primer período tienden a disminuir. La razón nuevamente está asociada al efecto que tiene tanto el ahorro como la reasignación de ocio entre períodos sobre la oferta de bienes no comerciables en el segundo período. 


\section{Gráfica 2.5}

Tasa de depreciación nominal

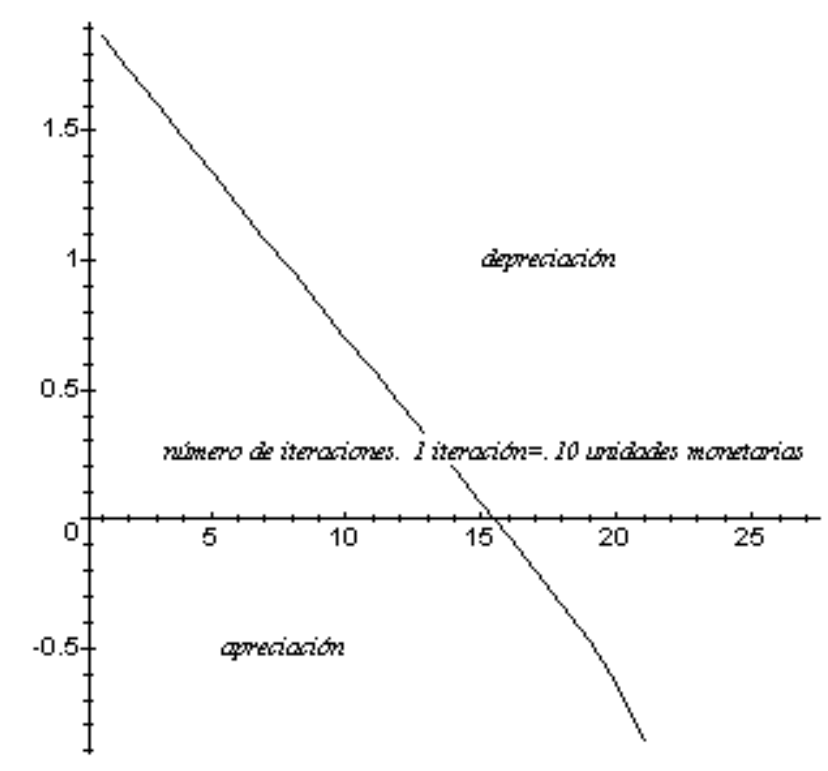

Hay que decir que por construcción del modelo, en la medida en que el sector financiero interno va creciendo, también van aumentando las operaciones internacionales y con ello los flujos de capitales del exterior. Es entonces un resultado del modelo el hecho de que el ahorro interno y el externo varíen en forma paralela. Es así que el ahorro interno coincide con la acumulación de activos internacionales en el primer período, y el mayor consumo en el período 2, con un déficit de cuanta corriente en el período terminal. 


\section{Gráfica 2.7}

Balanza comercial $(t=1)$

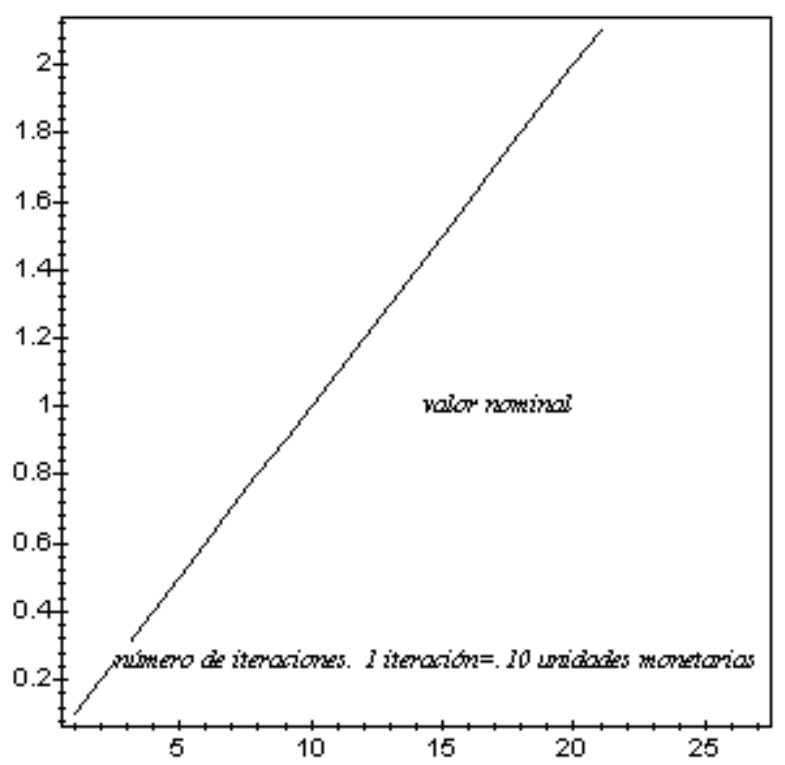

Es prudente prevenir al lector de que esta estructura de ahorro intertemporal depende crucialmente del valor de los parámetros y de la tecnología disponible, de tal suerte que para valores de $\theta<0.5$ podríamos ver déficit externos en el primer período y superávits en el segundo.

Por último, hay que enfatizar que los resultados que aparecen en las gráficas corresponden a niveles de profundización financiera menores al óptimo, por lo que, más allá de tal nivel, la política monetaria es totalmente neutral impactando exclusivamente el nivel de precios y el tipo de cambio nominal sin consecuencias sobre otros precios relativos, y consiguientemente sobre los patrones de asignación de recursos. 


\section{Gráfica 2.8}

Balanza comercial $(\mathbf{t}=\mathbf{2})$

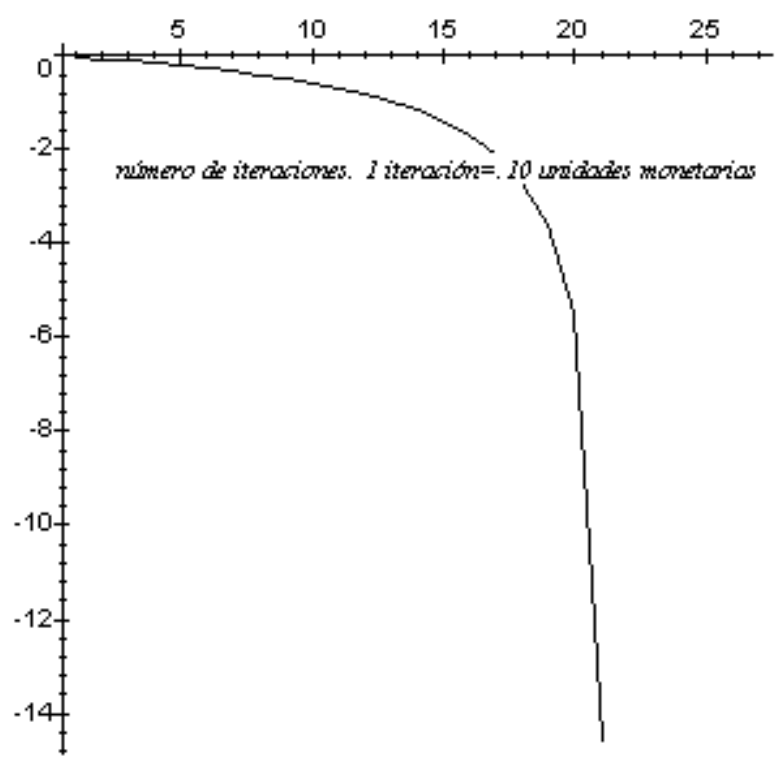

3. Los alcances de la política monetaria en México: hechos estilizados

Hemos visto que tanto en la literatura como desde un punto de vista conceptual la política monetaria tiene un papel importante en el proceso de desarrollo económico. Sin embargo, también tiene limitaciones muy importantes.

Por un lado, el desarrollo del sistema financiero es importante para facilitar una asignación de recursos adecuada, entre actividades y a través del tiempo. Pero poco es lo que puede hacerse para expandir arbitrariamente la economía o influir sobre el tipo de cambio real.

El Banco de México, a través de diversos documentos, ha manifestado su acuerdo con estas ideas. Por ejemplo, en el Informe sobre la Política Monetaria correspondiente al primer semestre de 1997, están presentes las dos ideas que han sido parte central de este documento; por un lado, la neutralidad de la política monetaria contracíclica y por el otro, el papel de la liquidez y del desarrollo de los mercados financieros para apoyar el crecimiento en el largo plazo:

"...Una de las principales lecciones que derivan tanto de la experiencia internacional, como de la propia, es que una política monetaria expansiva carece de capacidad para estimular el crecimiento y el empleo, salvo en condiciones muy especiales ..." , "...En otras palabras, no existe una relación positiva y estable entre inflación y empleo susceptible de ser explotada para mejorar el desempeñ o de la economía..." (pp.138).

"...el programa monetario para 1997 establece que el instituto emisor, como regla general, ajustará diariamente la oferta de dinero primario en forma en que dicha oferta corresponda a 
la demanda de base monetaria..." (pp.66)

Ambas afirmaciones son de gran trascendencia. Sin embargo, a juzgar por el debate que prevalece en las discusiones públicas sobre el papel de la política monetaria, aún puede resultar muy útil revisar la información disponible para determinar hasta qué grado los postulados de la teoría y las afirmaciones del instituto central corresponden adecuadamente a la realidad de México.

\section{Hechos estilizados}

Como primera aproximación seleccionamos dos agregados monetarios; uno estrecho, como es billetes y monedas en circulación y el agregado monetario más amplio. Paralelamente se escogieron las variables del sector real que pensamos son las más importantes desde el punto de vista de la discusión como son el PIB, el tipo de cambio real, la inversión y otras variables reales, el índice de precios al consumidor y las tasas de interés nominales. Todas las series tienen frecuencia trimestral y fueron obtenidas de la base SINIEE de Banco de México para el período de 1980-I a 1997-II.

1. Agregados monetarios y el nivel general de precios

Las gráficas de dispersión 3.1. y 3.2, muestran la relación que existe entre el logaritmo natural del nivel de precios medido por el Indice Nacional de Precios al Consumidor y los billetes y monedas en circulación y M4, respectivamente. Lo que ahí aparece habla por sí mismo al observarse una relación muy directa y estable entre ambas variables durante los 17 añ os de la muestra.

\section{Gráfica 3.1}

Logaritmo de billetes y monedas vs logaritmo del INPC

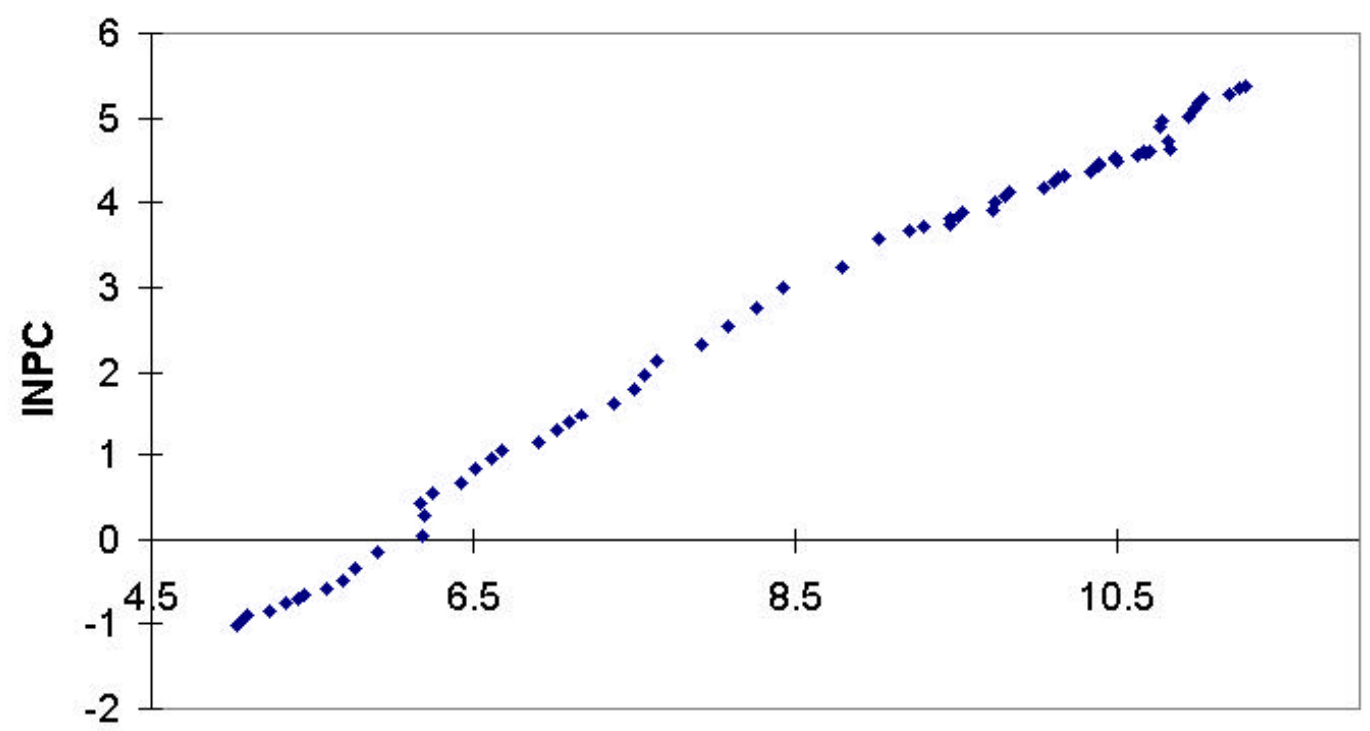

ByM 


\section{Gráfica 3.2}

\section{Logaritmo de M4 vs logaritmo del INPC}

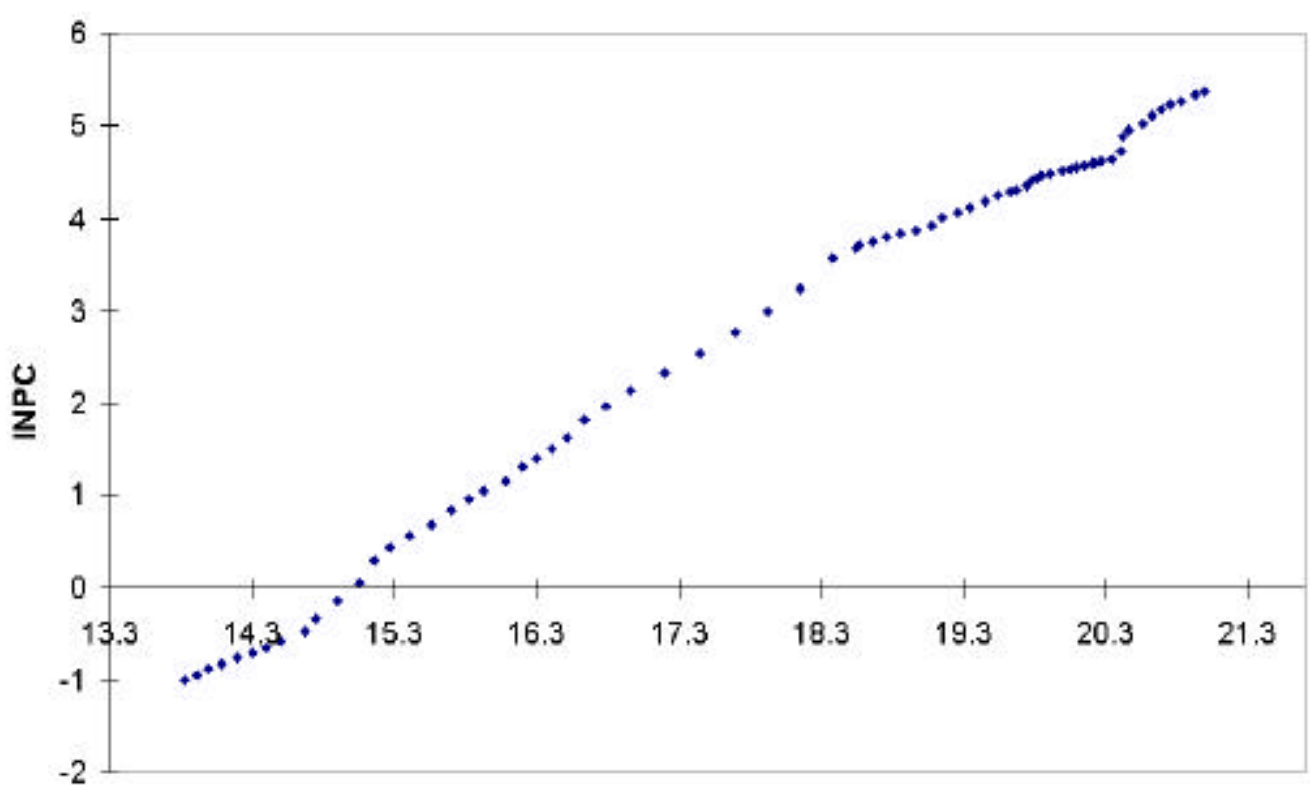

M4

2. Agregados monetarios y tasas de interés nominales

La relación entre los agregados monetarios y las tasas de interés nominales se muestra en las gráficas 3.3. y 3.4. En este caso, se observa una mayor dispersión que en el caso anterior. En el análisis estadístico que se presenta más adelante se harán pruebas de cointegración entre las variables que aparecen aquí graficadas; sin embargo, por inspección se antoja pensar que el comportamiento de las tasas nominales, aún si existiera alguna conexión con los agregados monetarios, no depende exclusivamente de ellos sino muy probablemente de un conjunto más amplio de factores que van desde las expectativas, la situación de las finanzas públicas, choques externos y cambios institucionales. Además parece presentarse un cambio estructural en la relación a partir de niveles en los que el logaritmo de billetes y monedas es superior a 9 y el de M4 superior a 18. 


\section{Gráfica 3.3}

Logaritmo de billetes y monedas vs tasa de CETES a 28 días

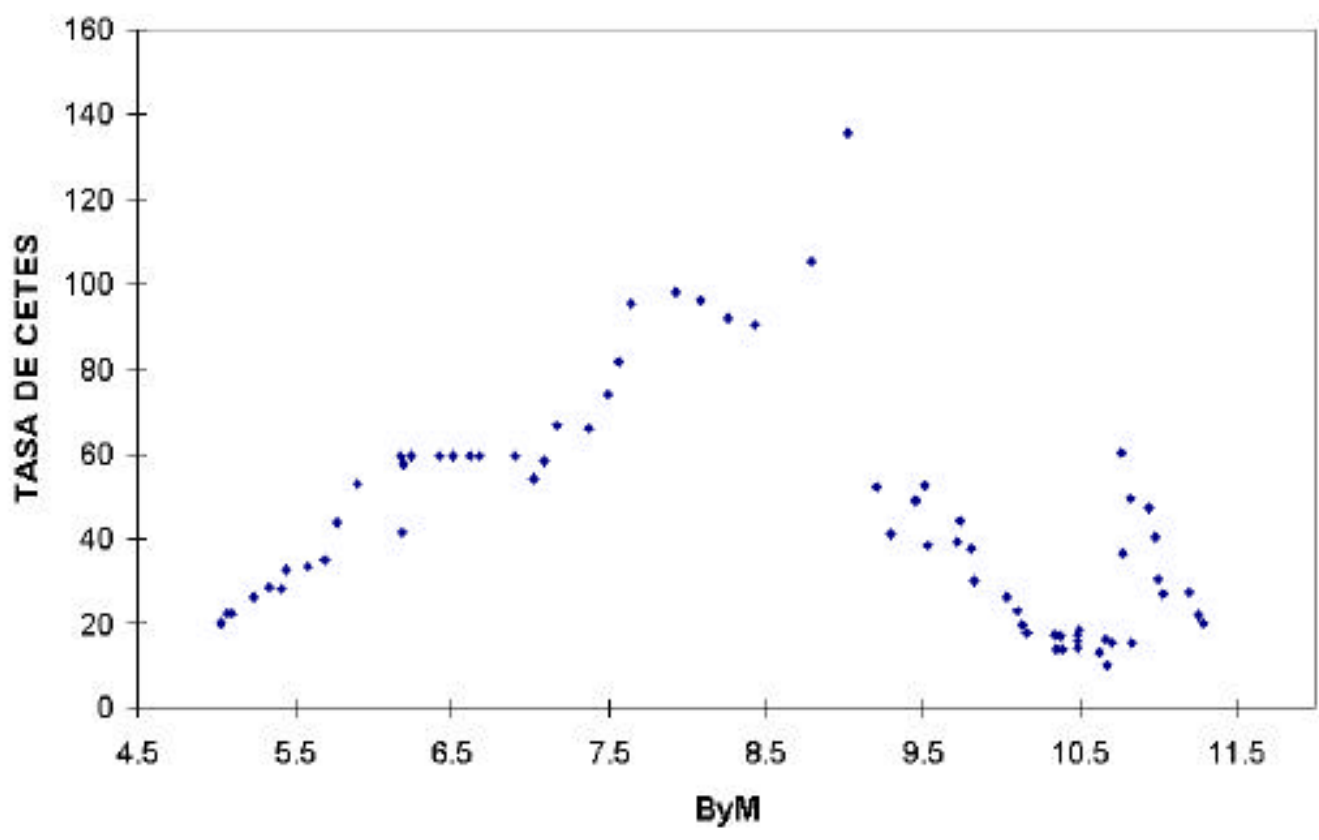

Gráfica 3.4

Logaritmo de M4 vs tasa de CETES a 28 días

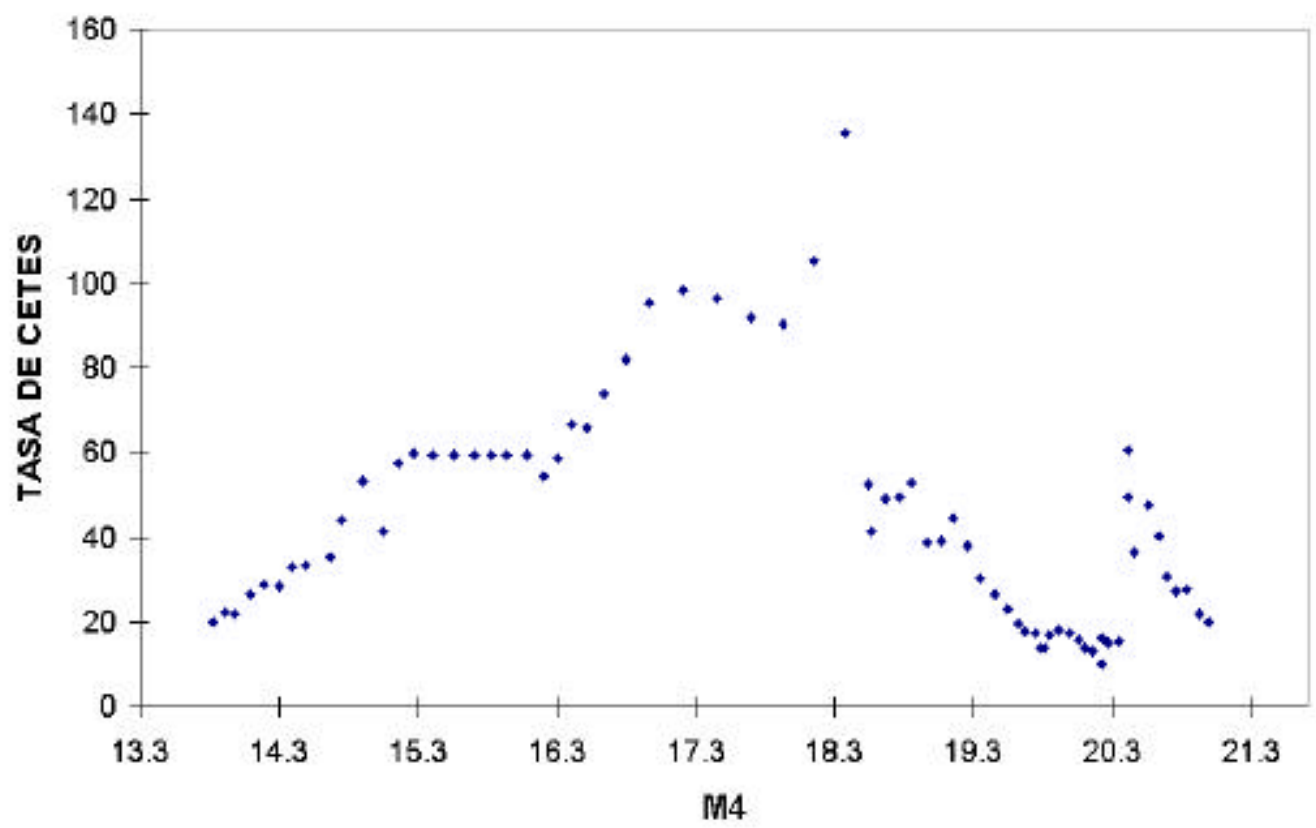


3. Agregados monetarios y PIB

Las gráficas 3.5 y 3.6 corresponden a la relación entre el logaritmo de los saldos nominales de billetes y monedas y de M4 contra PIB en términos reales. Destaca la ausencia aparente de una relación de cointegración entre variables nominales y reales. En estas gráficas parece haber dos tramos, en primer lugar, para niveles bajos de saldos monetarios parece que el nivel del PIB real se mantiene más o menos constante mientras la cantidad de dinero se incrementa. Por el contrario, cuando la cantidad nominal de dinero se estabiliza en valores relativamente altos, es el PIB el que muestra una mayor dispersión. En cualquier caso, resultaría muy difícil argumentar que pudiera existir alguna combinación lineal entre los citados agregados y el nivel de actividad (ambos expresados como logaritmo natural) que diese como resultado una serie estacionaria. (Hay que insistir en que aun si existiera una gráfica en la cual se observara una relación lineal perfecta entre el PIB real y los agregados monetarios en términos nominales, ello no implicaría necesariamente que dichas variables estuvieran cointegradas. Bastaría, por ejemplo, que sus ordenes de integración sean diferentes para que tal fuera el caso.)

\section{Gráfica 3.5}

Logaritmo de billetes y monedas vs logaritmo de PIB real

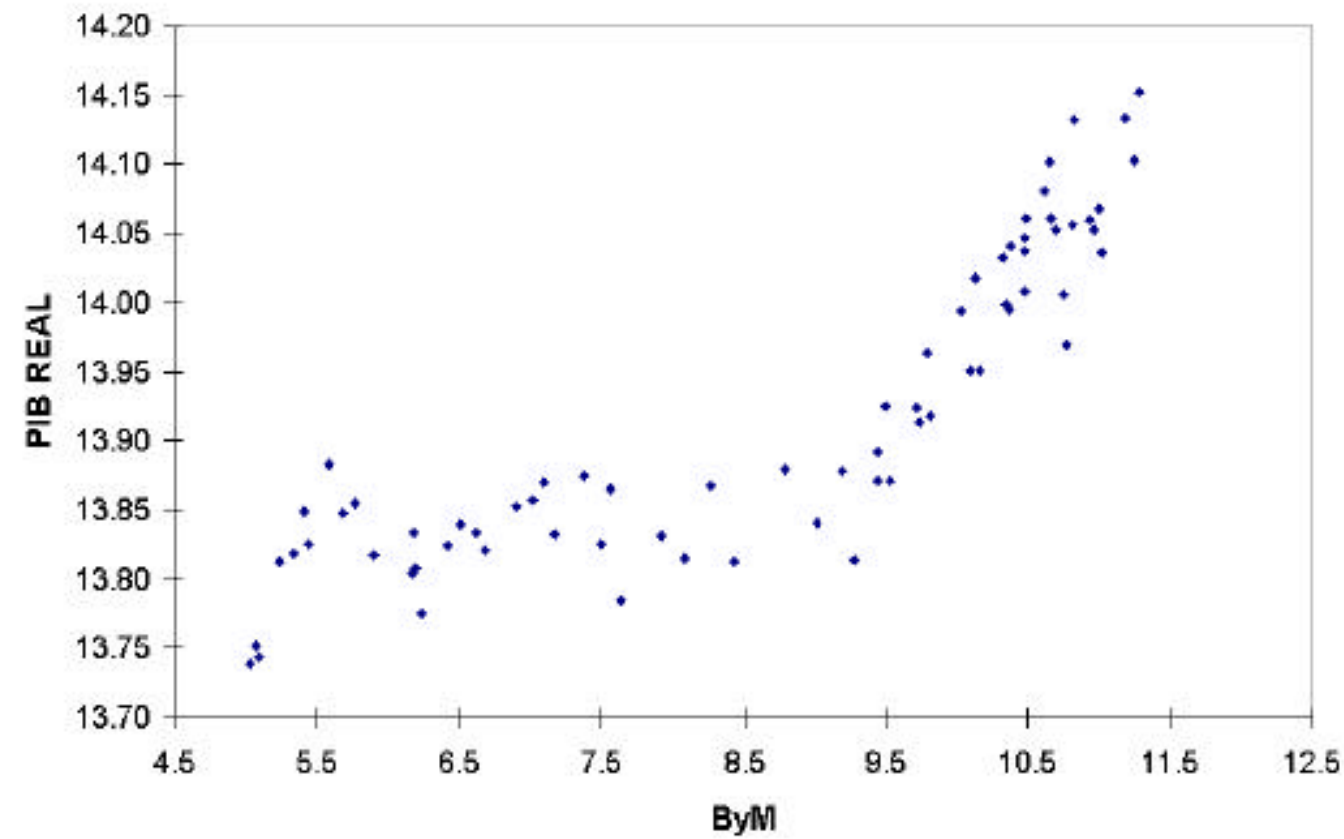




\section{Gráfica 3.6}

Logaritmo de M4 vs logaritmo del PIB

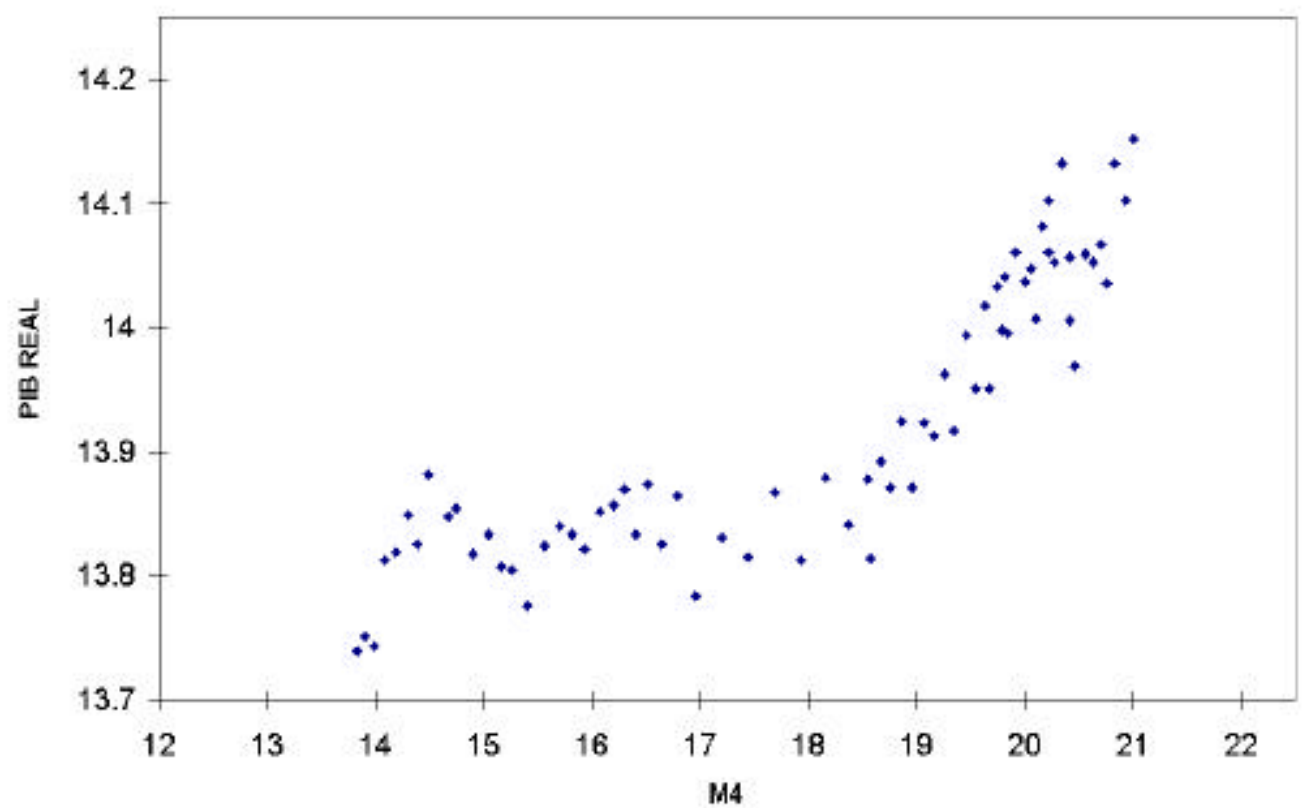

4. Agregados monetarios y tipo de cambio real

Nuevamente en este caso resulta muy difícil establecer una relación estable entre los agregados nominales y una variable real como es el tipo de cambio multilateral. Las gráficas muestran amplias fluctuaciones del logaritmo del tipo de cambio real a las que no corresponden fluctuaciones igualmente notables del medio circulante y viceversa. Conviene subrayar que esta característica se observa bajo los distintos regímenes cambiarios que han estado en operación en este país, pasando desde el deslizamiento predeterminado, un esquema de bandas y la libre flotación. 


\section{Gráfica 3.7}

Logaritmo de billetes y monedas vs logaritmo del tipo de cambio real

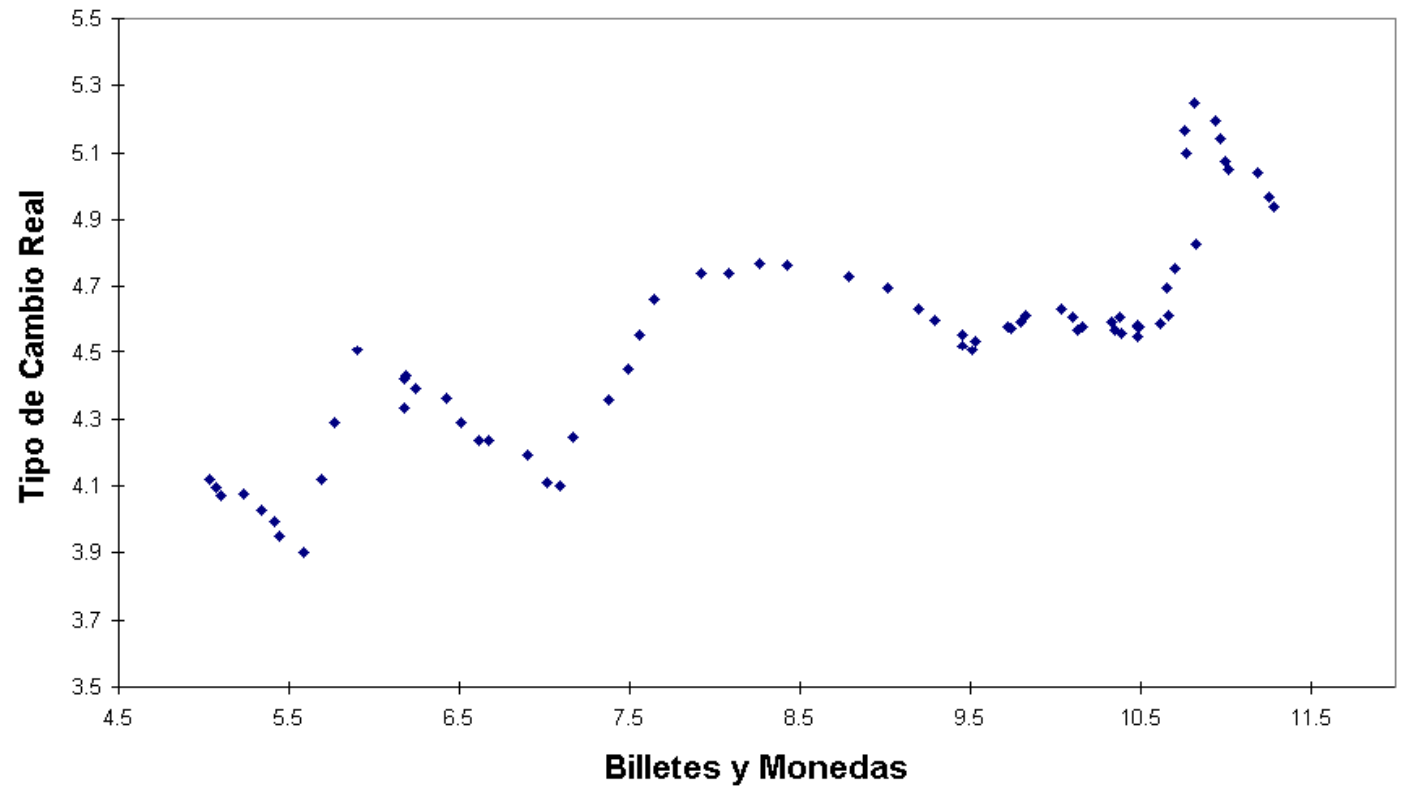

A manera de síntesis valdría la pena resaltar el enorme contraste que existe entre las gráficas que relacionan a los agregados monetarios con el nivel de precios y las demás. En las primeras queda poco lugar a dudas de que la relación que prevalece entre variables es fuerte y estable, mientras que en las otras uno se ve obligado a concluir que los procesos asociados a la determinación de las variables reales son complejos y cuyo comportamiento muy difícilmente puede ser influido de manera consistente únicamente mediante cambios en los niveles de las variables monetarias. 


\section{Gráfica 3.8}

Logaritmo de M4 vs logaritmo del tipo de cambio real

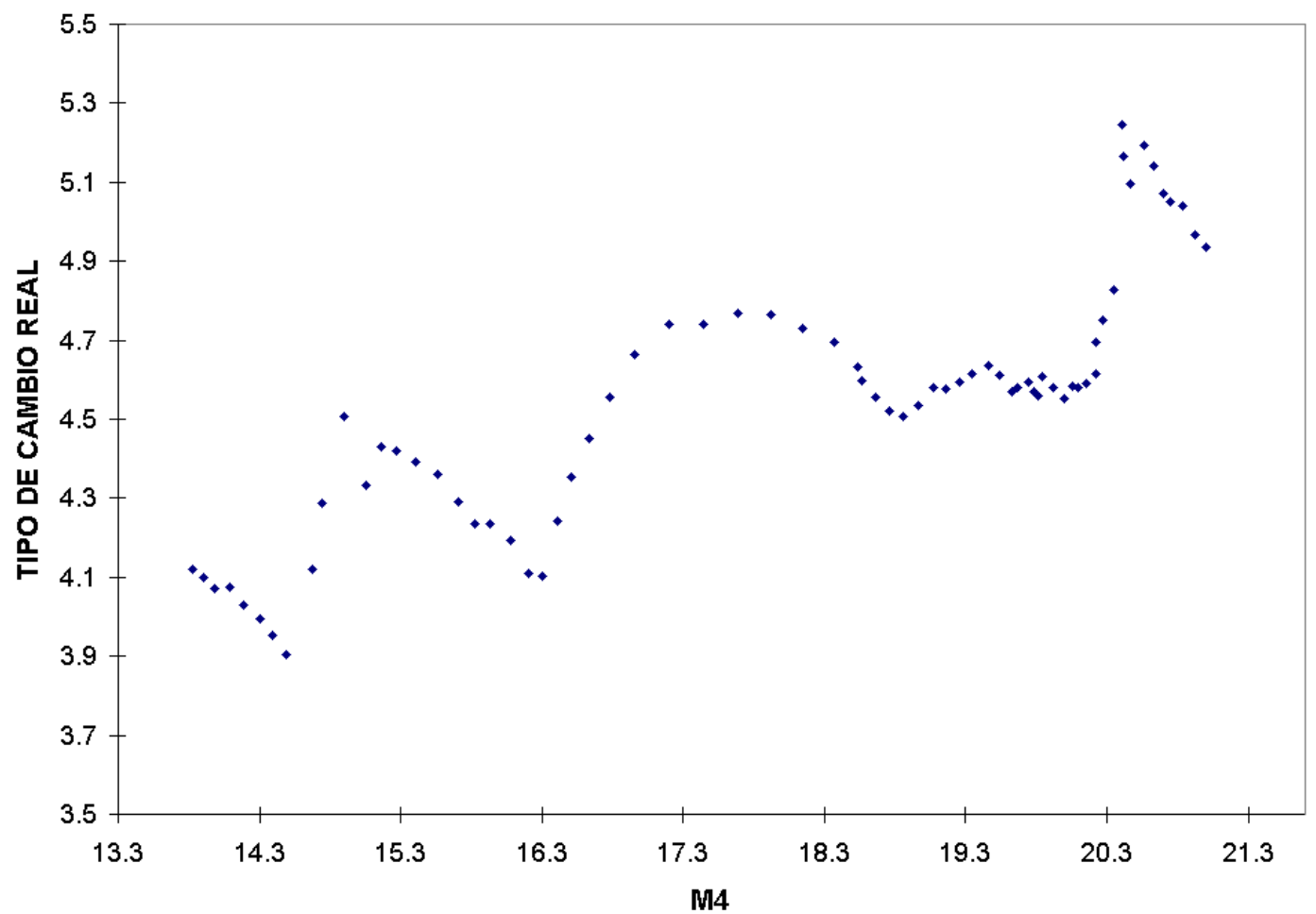

\section{Pruebas de cointegración}

Una manera de formalizar los comentarios que surgieron de la inspección de las gráficas, precedentes consiste en aplicar pruebas de cointegración entre agregados monetarios y las variables nominales y reales comentadas y a las cuales se incorpora la formación bruta de capital fijo y la cuenta corriente.

Antes de entrar en los detalles estadísticos, quisiéramos detenernos un momento a explicar con mayor precisión lo que estamos buscando. Como dijimos en los primeros párrafos de este documento, no dudamos que la política monetaria, junto con otros instrumentos, pueda formar parte de un paquete de medidas orientadas a promover la actividad, el empleo y la competitividad. Sin embargo, también queremos insistir en que cuando un instrumento tiene una relación fuerte y estable con alguna de las variables endógenas de la economía, tal instrumento debe concentrarse en influir sobre aquélla.

El análisis de cointegración que realizamos es predominantemente bivariado. Esto quiere decir que si no encontramos una relación de cointegración entre los agregados monetarios y alguna otra variable, no estamos descartando que pueda existir alguna relación de largo plazo entre un paquete de instrumentos y una variable objetivo como sería el PIB. Sin embargo, lo que sí se estaría desprendiendo es que difícilmente podría afirmarse que por sí sola la política monetaria estaría en condiciones de influir en el comportamiento de la variable objetivo.

Pensamos que ésta es una forma de interpretar el principio de la "clasificación del mercado más eficaz", según el cual desde el punto de vista de la política monetaria la tarea de las autoridades consistiría en encontrar aquéllas variables sobre las 
cuales su incidencia es más clara y eficaz a la hora de establecer sus metas. En términos de esta sección lo antes dicho equivaldría a encontrar aquellas variables con las que los agregados monetarios, que las autoridades puedan controlar, que tengan una relación de cointegración

Entrando en materia, y como es usual, el punto de partida consiste en determinar el orden de integración de las variables en cuestión mediante las correspondientes pruebas de raíces unitarias.

El cuadro 3.2. presenta las pruebas de Dickey-Fuller Aumentada (ADF) y de Phillips Perron (Como referencia de los métodos aplicados en esta sección se sugiere consultar Harris [1995].) (PP) para valores reales y nominales del logaritmo de billetes y monedas en circulación, logaritmo de M4, logaritmo del tipo de cambio, logaritmo de los ingresos de la cuenta corriente, logaritmo de los egresos de la cuenta corriente, logaritmo del saldo en la cuenta corriente (normalizado para evitar valores negativos), logaritmo del PIB y logaritmo de la formación bruta de capital fijo y la tasa de interés. (Logaritmo se refiere a logaritmo natural.)

Cada una de las celdas contiene el resultado de la pruebas para un $5 \%$ de significancia. Las últimas dos columnas muestran el orden de integración que se concluye tiene cada una de las variables. Hay que notar que las celdas en que se reporta la existencia de raíces unitarias con un "SI", corresponden a los casos en los que las pruebas respectivas llevan siempre a tal resultado habiendo especificado el modelo para cada variable con tendencia y constante, con constante y sin constante, según se presenta enseguida para la prueba ADF (Para la prueba PP se utilizó la misma estructura de rezagos.):

$$
\begin{aligned}
& \mathscr{A}_{t}=a_{2} z_{t-1}+a_{3} \mathscr{y}_{t-1}+c \\
& \mathscr{A}_{t}=a_{0}+a_{2} z_{t-1}+a_{3} \mathscr{z}_{t-1}+c \\
& \mathscr{A}_{t}=a_{0}+a_{1} t+a_{2} z_{t-1}+a_{3} \mathscr{A}_{t-1}+c
\end{aligned}
$$

donde $z_{t}$ se refiere al logaritmo de la variable sometida a la prueba. (En el caso de billetes y monedas se amplió el número de rezagos de la variable $\Delta \mathrm{z}_{\mathrm{t}}$ - $\mathrm{i}$ hasta $\mathrm{i}=4$ en virtud de que para menos rezagos aparecían contradicciones entre las pruebas de raíces unitarias y las de cointegración, mismas que desaparecieron al incluirse tal número de rezagos.) 


\section{Cuadro 3.1.1}

Características de las series utilizadas

\begin{tabular}{|c|c|}
\hline Variable & Unidades \\
\hline Indice nacional de Precios al Consumidor (INPC). & Base $1994=100$ \\
\hline Billetes y monedas en poder del público. & Saldos en millones de pesos corrientes. \\
\hline Agregado monetario amplio M4. & Saldos en miles de pesos corrientes. \\
\hline $\begin{array}{l}\text { Billetes y monedas en poder del público en } \\
\text { términos reales. }\end{array}$ & $\begin{array}{l}\text { Billetes y monedas en poder del público entre INPC. } \\
\text { Saldos en millones de pesos constantes. }\end{array}$ \\
\hline $\begin{array}{l}\text { Agregado monetario amplio M4 en términos } \\
\text { reales. }\end{array}$ & $\begin{array}{l}\text { M4 entre INPC } \\
\text { Saldos en miles de pesos constantes. }\end{array}$ \\
\hline Cuenta Corriente Ingresos. & Flujos en miles de millones de pesos corrientes. \\
\hline Cuenta Corriente Egresos. & Flujos en miles de millones de pesos corrientes. \\
\hline Balance de la Cuenta Corriente. & $\begin{array}{l}\text { Cuenta Corriente Ingresos - Cuenta Corriente Egresos. } \\
\text { Flujos en miles de millones de pesos corrientes. }\end{array}$ \\
\hline CETES a 28 días. & $\begin{array}{l}\text { Del primer trimestre de } 1980 \text { al tercer trimestre de } 1982 \\
\text { se utiliza la tasa de CETES a } 91 \text { días. }\end{array}$ \\
\hline Producto Interno Bruto Real. & Millones de pesos constantes $(1993=100)$. \\
\hline Formación Bruta de capital Fijo. & $\begin{array}{l}\text { Indice de volumen de la inversión fija bruta. } \\
\text { Pesos constantes }(1980=100) .\end{array}$ \\
\hline $\begin{array}{l}\text { Indice de Tipo de Cambio Real Efectivo Multilateral } \\
\text { (canasta con } 133 \text { países). }\end{array}$ & Número índice (1994=100). \\
\hline Tipo de Cambio Nominal. & $\begin{array}{l}\text { Tipo de cambio promedio de cotizaciones diarias. } \\
\text { Pesos por dólar. }\end{array}$ \\
\hline
\end{tabular}

Como se observa en el cuadro 3.1.2, es importante hacer notar que los agregados monetarios expresados en términos nominales son de orden I(2), situación que sólo es compartida por el Indice Nacional de Precios al Consumidor y el tipo de cambio nominal. En contraste, todas las demás variables son de orden I(1). Ello permite establecer que por construcción, sólo podría pensarse en una relación de cointegración (bi-variada) entre precios y agregados nominales.

Las pruebas de cointegración de Johansen se elaboraron a partir de modelos VAR de 6 rezagos con y sin tendencia y constante de regresión. (Los cálculos se efectuaron con E-Views, aplicando la rutina preestablecida en este paquete econométrico. ) 
Cuadro 3.1.2.

Pruebas de Raíces Unitarias

( $5 \%$ de significancia)

\begin{tabular}{|c|c|c|c|c|c|c|c|c|}
\hline & \multicolumn{2}{|c|}{ NIVELES } & \multicolumn{2}{|c|}{$\begin{array}{c}\text { PRIMERAS } \\
\text { DIFERENCI } \\
\text { AS }\end{array}$} & \multicolumn{2}{|c|}{$\begin{array}{c}\text { SEGUNDAS } \\
\text { DIFERENCI } \\
\text { AS }\end{array}$} & \multicolumn{2}{|c|}{$\begin{array}{l}\text { ORDEN DE } \\
\text { INTEGRACI } \\
\text { ON }\end{array}$} \\
\hline & ADF & PP & ADF & $\mathrm{PP}$ & ADF & PP & ADF & PP \\
\hline \multicolumn{9}{|c|}{ AGREGADOS MONETARIOS } \\
\hline Billetes y Monedas & NO & NO & NO & $\mathrm{SI}$ & SI & & $1(2)$ & $I(1)$ \\
\hline M4 & NO & NO & NO & NO & SI & $\mathrm{SI}$ & $1(2)$ & $\mathrm{I}(2)$ \\
\hline ByM real & NO & NO & SI & $\mathrm{SI}$ & & & $1(1)$ & $\mathrm{I}(1)$ \\
\hline M4 real & NO & NO & SI & $\mathrm{SI}$ & & & $1(1)$ & $I(1)$ \\
\hline \multicolumn{9}{|c|}{ VARI ABLES NOMI NALES } \\
\hline Indice de precios & NO & NO & NO & NO & SI & $\mathrm{SI}$ & $1(2)$ & $\mathrm{I}(2)$ \\
\hline Tipo de Cambio & NO & NO & NO & $\mathrm{SI}$ & SI & & $1(2)$ & $\mathrm{I}(1)$ \\
\hline Cta.Corr. ingresos & NO & NO & NO & $\mathrm{SI}$ & SI & & $1(2)$ & $I(1)$ \\
\hline Cta.Corr. egresos & NO & NO & NO & $\mathrm{SI}$ & SI & & $1(2)$ & $\mathrm{I}(1)$ \\
\hline Cta.Corr. balance & NO & NO & SI & $\mathrm{SI}$ & & & $1(1)$ & $\mathrm{I}(1)$ \\
\hline Tasa de Interés & NO & NO & SI & $\mathrm{SI}$ & & & $1(1)$ & $\mathrm{I}(1)$ \\
\hline \multicolumn{9}{|c|}{ VARI ABLES REALES } \\
\hline PIB & NO & NO & SI & $\mathrm{SI}$ & & & $1(1)$ & $\mathrm{I}(1)$ \\
\hline Inversión & NO & NO & SI & $\mathrm{SI}$ & & & $1(1)$ & $I(1)$ \\
\hline T. de Cambio Real & NO & NO & SI & $\mathrm{SI}$ & & & $I(1)$ & $I(1)$ \\
\hline
\end{tabular}

Adicionalmente, se trabajó con variables reales y variables nominales en pares y en grupos de variables. 


\section{Cuadro 3.2}

Pruebas de Cointegración de Johansen

( $5 \%$ de significancia)

\begin{tabular}{|c|c|c|c|c|c|c|c|c|}
\hline & \multicolumn{2}{|c|}{$\begin{array}{r}\text { Billetes y } \\
\text { Monedas } \\
(\text { no } \\
\text { min } \\
\text { al) }\end{array}$} & \multicolumn{2}{|c|}{$\begin{array}{l}\text { M4 } \\
\quad \begin{array}{c}\text { ( no } \\
\text { min } \\
\text { al) }\end{array}\end{array}$} & \multicolumn{2}{|c|}{$\begin{array}{c}\text { Billetes y } \\
\text { Monedas } \\
\text { ( rea } \\
\text { I) }\end{array}$} & \multicolumn{2}{|c|}{$\begin{array}{l}\text { M4 } \\
\quad \begin{array}{l}\text { (rea } \\
\text { I) }\end{array}\end{array}$} \\
\hline & (a) & (b) & (a) & (b) & (a) & (b) & (a) & (b) \\
\hline \multicolumn{9}{|c|}{ VARI ABLES NOMI NALES } \\
\hline Indice de precios & NO & $*$ & SI & $*$ & NO & $*$ & SI & $*$ \\
\hline Tipo de Cambio & SI & * & SI & $*$ & SI & $*$ & $*$ & $*$ \\
\hline CC balance & NO & NO & NO & NO & NO & NO & NO & NO \\
\hline Tasa de Interés & SI & $*$ & $\mathrm{SI}$ & $*$ & NO & NO & NO & NO \\
\hline \multicolumn{9}{|c|}{ VARI ABLES REALES } \\
\hline PIB & $*$ & $*$ & $*$ & * & $*$ & NO & NO & NO \\
\hline Inversión & $*$ & $*$ & $*$ & * & NO & NO & NO & SI \\
\hline TCR & NO & NO & NO & NO & SI & $*$ & NO & NO \\
\hline \multicolumn{9}{|l|}{ GRUPOS } \\
\hline$i, p$ & $\begin{array}{c}\text { SI } \\
\rho=1\end{array}$ & * & $\begin{array}{c}\text { SI } \\
\rho=1\end{array}$ & $*$ & NO & NO & NO & $\begin{array}{c}\text { SI } \\
\rho=1\end{array}$ \\
\hline PIB, y & $*$ & NO & $\begin{array}{c}\text { SI } \\
\rho=2\end{array}$ & NO & $\begin{array}{c}\mathrm{SI} \\
\rho=1\end{array}$ & NO & NO & NO \\
\hline PIB, TCR, INV. P. & $*$ & $*$ & $*$ & $*$ & $\underset{\rho=1}{S I}$ & $\underset{\rho=1}{S I}$ & $\begin{array}{c}\mathrm{SI} \\
\rho=2\end{array}$ & $\underset{\rho=1}{S I}$ \\
\hline
\end{tabular}

I a No incluye una tendencia determinística en los datos ni tampoco ordenada al origen.

$\backslash$ b Incluye una tendencia determinística en los datos y ordenada al origen.

I* E-Views reporta un rango de cointegración superior al máximo posible. Esto podría indicar dos cosas diferentes: (i) las variables son estacionarias y (ii) de no ser así, existe un problema de especificación, que no permite concluir sí existe cointegración.

$\rho$ corresponde al número de vectores de cointegración encontrados

Así vemos que los saldos de billetes y monedas en circulación sí cointegran con el tipo de cambio nominal y la tasa de interés, así como con el grupo construído por la tasa de interés nominal y el nivel de precios. Por su parte, mientras que M4 cointegra con el índice de precios, el tipo de cambio nominal y las tasas de interés. Al 
mismo tiempo no encontramos relación de cointegración alguna entre las variables monetarias en términos nominales y las variables de sector real, excepto cuando aparecen en grupo como es el caso de M4, la tasa de interés nominal y el PIB.

Estos resultados son notablemente congruentes con la percepción que las autoridades manifestaron en su Informe sobre la Política Monetaria citado anteriormente, así como con la hipótesis de neutralidad discutida a lo largo de este documento.

Quedaría por mencionar que la relación de cointegración que aparece entre los agregados monetarios en términos reales y grupos de variables nominales y reales, denotan la posible existencia de funciones de demanda de dinero estables (Gaytán y Rocha [1996].), situaciones que de hecho sirven al banco central para hacer su programación financiera en función de metas de crecimiento e inflación, más que con el propósito de influir directamente en el nivel de actividad y empleo. 


\section{Conclusiones}

En estos momentos, en los que existe un fuerte debate sobre el papel de la política económica en el desarrollo nacional, resulta fundamental tener muy claro cuáles son los alcances de cada herramienta. La literatura sugiere que la política monetaria tiene su mayor ventaja cuando se enfoca hacia el objetivo de procurar la estabilidad de los precios.

En países en vías de desarrollo, el ordenado crecimiento del sector financiero es también importante, en la medida en que ello permita la más adecuada asignación de recursos entre ahorradores e inversionistas. Podríamos decir entonces que una política monetaria que proporcione la liquidez que necesita la economía para efectuar las transacciones corrientes e intertemporales asociadas con la dinámica propia del sector real, y que además busque el moderado crecimiento de los precios, es la más adecuada. Este razonamiento que se desprende de los puntos de vista contenidos en numerosos artículos teóricos y econométricos de la experiencia internacional, encuentra un claro sustento en la experiencia mexicana de los últimos 17 añ os. 


\section{Apéndice 1. Rutina para la determinación de la cantidad óptima de dinero}

\section{En el presente apéndice se proporciona un algoritmo para realizar una rutina en MapleV Release 4 que permite obtener la cantidad óptima de dinero para una economía cerrada.}

restart;

utilidad: $=($ consumo1, consumo2, trabajo1, trabajo2) theta*ln(consu mo1^alpha*(24-trabajo1)^(1-alpha)) +(1theta)* In (consumo2^alpha *(24-trabajo2)^(1-alpha)):\# Comportamiento de las familias.

restriccion: $=($ trabajo1, trabajo2, consumo1, consumo2) - precio1 *consumo1 +precio2*consumo2-precio1*salario1*trabajo1precio1 *ganancias1-precio2*salario2*trabajo2precio2*ganancias2: ut riccion (trabajo1, trabajo2, consumo1, consumo2)) :

$1 c 1:=($ consumo1, consumo2, trabajo1, trabajo2, lambda $)-$ diff(lagran

giano (consumo1, consumo2, trabajo1, trabajo2, lambda), consumo1 ) : lc2:=(consumo1, consumo2, trabajo1, trabajo2, lambda) diff (lagrangia

no (consumo1, consumo2, trabajo1, trabajo2, lambda), consumo2) : 1 t1:= (consumo1, consumo2, trabajo1, trabajo2, lambda $)-$

diff (lagrangiano(

consumo1, consumo2, trabajo1, trabajo2, lambda), trabajo1) : 1t2:

$=($ consumo1, consumo2, trabajo1, trabajo2, lambda $)-$

diff (lagrangiano (con

sumo1, consumo2, trabajo1, trabajo2, lambda), trabajo2 $): 11:=($ co nsumo1, consumo2, trabajo1, trabajo2, lambda)-

diff (lagrangiano (consu

mo1, consumo2, trabajo1, trabajo2, lambda), lambda) :

solucion_familias:=solve $(\{1 c 1$ (consumo1, consumo2, trabajo1, t raba jo2, lambda) =0, lc2 (consumo1, consumo2, trabajo1, trabajo2, lambda) $=0$,

lt1 (consumo1, consumo2, trabajo1, trabajo2, lambda) =0, lt2 (cons umo1, consumo2, trabajo1, trabajo2, lambda)=0, 11 (consumo1, cons umo2, trabajo1, trabajo2, lambda) $=0\}$, \{consumo1, consumo2, traba jo1, trabajo2, lambda\}) :assign (solucion_familias); \#Condicion es de ler orden.

dembienes1:=unapply (consumo1, precio1,precio2, salario1, sala rio2, ganancias1, ganancias2):\# Demanda de bienes en el ler período.

dembienes 2 :=unapply (consumo2, precio1, precio2, salario1, sala rio2, ganancias1, ganancias2): \# Demanda de bienes en el 2do período.

oftrabajo1:=unapply (trabajo1, precio1, precio2, salario1, sala rio2, ganancias1,ganancias2): \# Oferta de trabajo en el ler período.

oftrabajo2:=unapply (trabajo2, precio1, precio2, salario1, sala rio2, ganancias1, ganancias2): Oferta de trabajo en el 2 do período.

demdinero:=(precio1, precio2, salario1, salario2, ganancias1,g 
anancias 2$)-$

precio1*salario1*oftrabajo1 (precio1,precio2, salario1, salario2, ganancias1, ganancias2) +precio1*ganancias1preciol*dem

bienes1 (precio1, precio2, salario1, salario2, ganancias1, ganan cias2) :\# Demanda de dinero.

trabajo1:='trabajo1' :trabajo2:='trabajo2' :consumo1 :='consu

mo1' :

consumo2:='consumo2' : dinero:='dinero' :\#Comportamiento

empresas.

produccion1:=trabajol-trabajo1^betal:\# Prod en el ler

período.

produccion2:=trabajo2-trabajo2^beta2:\# Prod en el 2do período.

beneficios1:=trabajo1-produccion1 (trabajo1) -

salario1*trabajo1: beneficios2:=trabajo2-

produccion2 (trabajo2)-salario2*trabajo2:

bt $1:=\operatorname{diff}($ beneficios1 (trabajo1), trabajo1) : bt $2:=\operatorname{diff}($ benefi cios2 (trabajo2), trabajo2) :primer_orden_1:=unapply (bt1, trab ajo1) :primer_orden_2:=unapply (bt2, trabajo2) :

solucion_empresa:=solve ( primer_orden_1 (trabajo1)=0,primer _orden_2 (trabajo2) =0, , trabajo1, trabajo2 $\}$ ) : assign (solucion -empresa):

demtrabajo1:=unapply (trabajo1, salario1) : demtrabajo2 :=unapp ly (trabajo2, salario2):\# Demandas de trabajo en cada período.

ganancias1:=salario1-produccion1 (demtrabajo1 (salario1)) salario1*demtrabajo1 (salario1) : ganancias2:=salario2-produ ccion2 (demtrabajo2 (salario2)) -

salario2*demtrabajo2 (salario2) :

ofbienes1:=salario1-

produccion1 (demtrabajo1 (salario1)) :ofbie nes2:=salario2produccion2 (demtrabajo2 (salario2)) :

trabajo1:='trabajo1' :

dinero: ='dinero' :

trabajo2:='trabajo2' :

excessbienes1:=(precio1,precio2, salario1, salario2) -

dembienes 1

(precio1, precio2, salario1, salario2, ganancias1 (salario1), ga

nancias2 (salario2)) +ofdinero/precio1-ofbienes1 (salario1):

excessbienes $2:=($ precio1, precio2, salario1, salario2) -

dembienes 2

(precio1, precio2, salario1, salario2, ganancias1 (salario1), ga

nancias2 (salario2)) -ofbienes2 (salario2)-ofdinero/precio2:

excesstrabajo1:=(precio1,precio2, salario1, salario2)-

demtrabajo 1 (salario1)-

oftrabajo1 (precio1, precio2, salario1, salario2, ganancias1

(salario1), ganancias2 (salario2)) :

excesstrabajo2:=(precio1, precio2, salario1, salario2)-

demtrabajo 2 (salario2)-

oftrabajo2 (precio1, precio2, salario1, salario2, ganancias1

(salario1), ganancias2 (salario2)) :

excessdinero:=(precio1, precio2, salario1, salario2) -

demdinero

(precio1, precio2, salario1, salario2, ganancias1 (salario1), ga 
nancias2 (salario2)) -ofdinero:

factor( (precio1*excessbienes1 (precio1,precio2, salario1, sal ario2) +precio2*excessbienes2 (precio1, precio2, salario1, sala rio2) +precio1*salario1*excesstrabajo1 (precio1, precio2, sala rio1, salario2) +precio2*salario2*excesstrabajo2 (precio1, pre cio2, salario1, salario2)

precio1:=1: \# Numerario.

salariol:='salariol' :

salario2:='salario2' :

precio2:='precio2' :

alpha:=parámetro1: beta1:=parámetro2: beta2:= parámetro3: theta:= parámetro4: escalon:=parámetro5:\# Se int. los valores.

ofdinero: $=0$ : equilibrio: $=\{1\}$ :

while nops(\{equilibrio\}minus conjuntovacio)0 do

ofdinero:=ofdinerotescalon:

equilibrio:=solve ( excesstrabajo1 (precio1, precio2, salario1 , salario2) =0, excesstrabajo2 (precio1, precio2, salario1, salar io2) $=0$, excessbienes 2 (precio1, precio2, salario1, salario2)=0, precio20, salario10, salario20\}, \{precio2, salario1, salario2\}) ; od:

ofdinero:=ofdinero-escalon:\# Cantidad óptima de dinero. , salario2) =0, excesstrabajo2 (precio1, precio2, salario1, salar io2) $=0$, excessbienes2 (precio1, precio2, salario1, salario2)=0, precio20, salario10, salario20\}, \{precio2, salario1, salario2\}) :assign(equilibrio):\# Se presentan las principales variables macroeconómicas.

PIB1:=ofbienes1 (salario1); PIB2:=ofbienes2 (salario2); empleo $1:=$ demtrabajo1 (salario1); empleo2:=demtrabajo2 (salario2); in flacion: $=($ precio2

precio1)/preciol;tasa_real:=(precio1/precio2) -1 ;

consumo_privado1:=dembienes1 (precio1, precio2, salario1, sala rio2, ganancias1 (salario1), ganancias2 (salario2)) ; consumo_pr ivado2:=dembienes2 (precio1, precio2, salario1, salario2, ganan cias1 (salario1), ganancias2 (salario2));

ahorro1:=PIB1-Consumo_privado1; ahorro2:=PIB2consumo_privado2;

Bienestar Social:=utilidad (consumo privado1, consumo_privad o2, demtrabajo1 (salario1), demtrabajo2 (salario2));

cantidad_optima_de_dinero:=ofdinero;

nivel_optimo_de_bienestar:=Bienestar_Social; 
Apéndice 2. Rutina para la determinación de la cantidad óptima de dinero ante la presencia del señoriaje

A continuación se presenta un algoritmo en MapleV Release 4 que permite obtener la cantidad óptima de dinero cuando se presentan tecnologías de almacenamiento alternativas, en este caso el impuesto inflacionario.

Debido a las similitudes con la rutina del apéndice 1 solamente se presentan las líneas que se deben sustituir.

- Sustituir la línea (3) por:

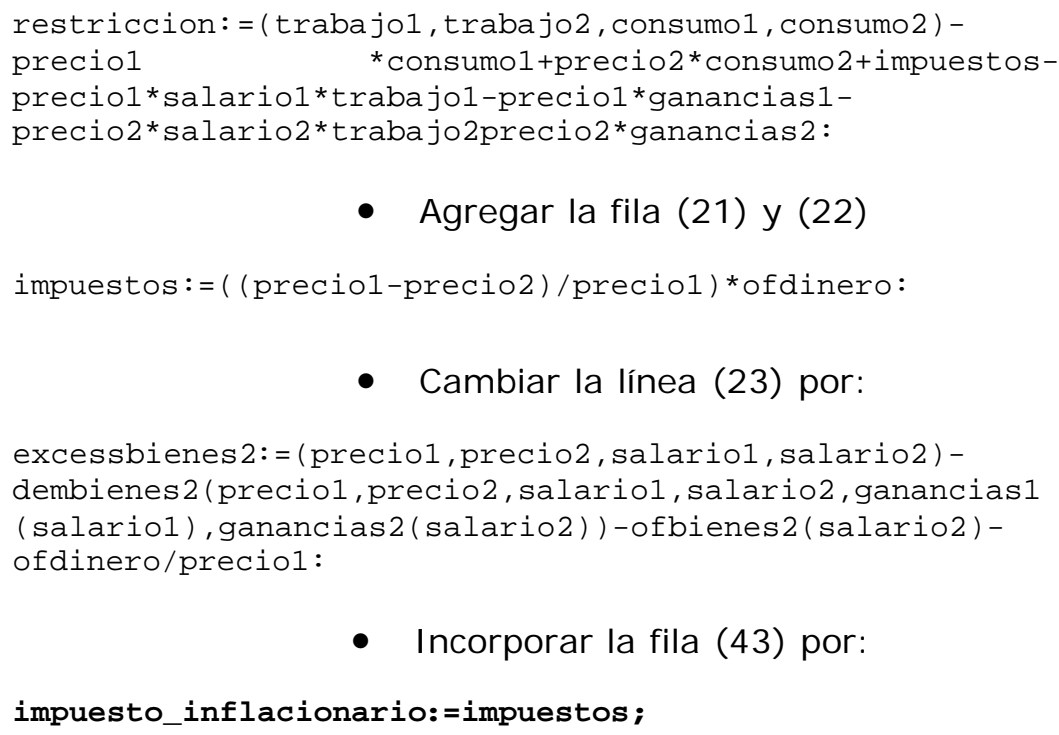

- Agregar la fila (21) y (22)

impuestos:=( (precio1-precio2)/precio1) *ofdinero:

- Cambiar la línea (23) por:

excessbienes $2:=($ precio1, precio2, salario1, salario2)-

dembienes2 (precio1, precio2, salario1, salario2, ganancias1

(salario1), ganancias2 (salario2)) -ofbienes2 (salario2) ofdinero/preciol:

- Incorporar la fila (43) por:

impuesto_inflacionario:=impuestos; 


\title{
Apéndice 3. Determinación de la cantidad de dinero óptima en economía abierta
}

\author{
La siguiente rutina proporciona los comandos necesarios para obtener las condiciones de \\ decisión óptimas en el caso de economía abierta.
}

utilidad: $=($ consumo1, consumo2, importaciones 1, importacione s2,trabajo1, trabajo2) -

theta*ln ( (consumo1^rho*importaciones1^(1-rho))

^alpha* (24-trabajo1)^(1-alpha)) +(1-

theta)* $\ln \left(\left(\right.\right.$ consumo $2^{\wedge}$ rho*im

rho ) )^alpha* $\left.(24-\text { trabajo2 })^{\wedge}(1-a l p h a)\right)$ :

restriccion:= (trabajo1, trabajo2, consumo1, consumo2, import aciones1, importaciones2) -

precio $1 *$ consumo $1+$ precio $2 *$ consumo $2+$ exr 1

*importaciones1+exr2*importaciones2-

precio1*salario1*trabajo1-ganancias1-precio2

*salario2*trabajo2-ganancias2:

lagrangiano: $=($ consumo 1, consumo 2 , importaciones 1 , importaci ones2, trabajo1, trabajo2, lambda) -

utilidad (consumo1, consumo2, importacio

nes1, importaciones2, trabajo1, trabajo2) +lambda* (restricci on (trabajo1, trabajo2, consumo1, consumo2, importaciones1, im portaciones2):

$1 \mathrm{c} 1:=($ consumo1, consumo2, importaciones 1, importaciones $2, \mathrm{tr}$ abajo1, trabajo2, lambda) -

diff (lagrangiano (consumo1, consumo2, importa

ciones1, importaciones2, trabajo1, trabajo2, lambda), consumo 1) : lc2:=(consumo1, consumo2, importaciones1, importaciones 2 , trabajo1, trabajo2, lambda)-

diff (lagrangiano (consumo1, consumo2, importacio

nes1, importaciones2, trabajo1, trabajo2, lambda), consumo2): $1 \mathrm{~m} 1:=($ consumo1, consumo2, importaciones 1 , importaciones $2, \mathrm{tr}$ abajo1, trabajo2, lambda)-

diff (lagrangiano (consumo1, consumo2, importaciones1, importaciones2, trabajo1, trabajo2, lambda), importaciones1) : $1 \mathrm{~m} 2:=$ (consumo1, consumo2, importaciones 1 , importaciones $2, t$ rabajo1, trabajo2, lambda)-

diff (lagrangiano (consumo1, consumo2, importaciones1, importaciones2, trabajo1, trabajo2, lambda), importaciones2) $:$ It $1:=$ (consumo1, consumo2, importaciones 1 , importaciones $2, t$ rabajo1, trabajo2, lambda) -

diff (lagrangiano (consumo1, consumo2, importaciones1,

importaciones2, trabajo1, trabajo2, lambda), trabajo1) : 1t2:= (consumo1, consumo2, importaciones1, importaciones2, trabajo 1 , trabajo2, lambda) -

diff (lagrangiano (consumo1, consumo2, importaciones1, import a

ciones2, trabajo1, trabajo2, lambda), trabajo2) : ll:=(consumo 1 , consumo2, importaciones1, importaciones 2, trabajo1, trabaj o2, lambda) -di

ff (lagrangiano (consumo1, consumo2, importaciones1, importac iones2, trabajo1, trabajo2, lambda), lambda) :

solucion_familias:=solve ( $\{$ lc1 (consumo1, consumo2, importac iones1, importaciones2, traba jo1, traba jo2, lambda) =0, lc2 (co nsumo1, consumo2, importaciones1, importaciones2, trabajo1, t rabajo2, lambda) $=0, \operatorname{lm} 1$ (consumo1, consumo2, importaciones $1, i$ 
mportaciones2, trabajo1, trabajo2, lambda) =0, $1 \mathrm{~m} 2$ (consumo1, c onsumo2, importaciones1, importaciones 2, trabajo1, trabajo2, lambda) $=0,1 t 1$ (consumo1, consumo2, importaciones 1 , importaci ones2, trabajo1, trabajo2, lambda) =0, 1 t2 (consumo1, consumo2, importaciones1, importaciones 2 , trabajo1, trabajo2, lambda) = 0,11 (consumo1, consumo2, importaciones1, importaciones 2 , tra bajo1, trabajo2, lambda) $=0\},\{$ consumo1, consumo2, importacion es1, importaciones2, traba jo1, traba jo2, lambda\}) : assign (sol ucion_familias);

dembienes 1 :=unapply (consumo1,precio1,precio2, salario1, sa lario2, ganancias1, ganancias2, exr1, exr2) :dembienes2:=unap ply (consumo2, precio1, precio2, salario1, salario2, ganancias 1,ganancias2, exr1, exr2) :\# Demanda de bienes en cada período.

demimport1:=unapply (importaciones1,precio1,precio2, salar io1, salario2, ganancias1, ganancias2, exr1, exr2) : demimport2 :=unapply (importaciones2, precio1, precio2, salario1, salari

o2, ganancias1,ganancias2, exr1, exr2):\# Demanda de importaciones en cada período.

oftrabajo1 :=unapply (trabajo1, precio1, precio2, salario1, sa lario2, ganancias1, ganancias2, exr1, exr2) : oftrabajo2:=unap ply (trabajo2, precio1, precio2, salario1, salario2, ganancias 1, ganancias2, exr1, exr2) :\# Oferta de trabajo en cada período.

trabajo1:='trabajo1' :trabajo2:='trabajo2' : consumo1 :='con sumo1': consumo2:='consumo2':dinero:='dinero' :

produccion $1:=$ trabajo11-

trabajo11^beta:produccion2 :=trabajo21-trabajo21^beta:\# Función de prod. en cada período.

exportacion1 :=trabajo12-

trabajo12^beta: exportacion $2:=t r a b a j o ~ 22-t r a b a j o 22^{\wedge}$ beta: \# Nivel de exportaciones en cada período

beneficios11:=trabajo11-precio1 *produccion1 (trabajo11)-

precio1 *salario1 *trabajo11:beneficios21:=trabajo21precio2*produccion 2(trabajo21)precio2*salario2*trabajo21:beneficios12:=trabajo 12exr1*exportacion1 (trabajo12)-precio1*salario1 *trabajo12: beneficios 22 :=trabajo22-exr2*exportacion2 (trabajo22)precio2* salario2*trabajo22:

beneficios1:=(trabajo11, trabajo12) -

beneficios11 (trabajo11) +be

neficios12 (trabajo12) : beneficios 2 :=(trabajo21, trabajo22)

-beneficios21 (trabajo21) +be neficios22 (trabajo22) :

$\mathrm{d} 11:=($ trabajo11, trabajo12) -

diff (beneficios1 (trabajo11, trabajo

12), trabajo11) : d12:=(trabajo11, trabajo12) -

diff (beneficios1 (tra

bajo11, trabajo12), trabajo12) : d21:=(trabajo21, trabajo22) diff

(beneficios2 (trabajo21, trabajo22), trabajo21) : d22:=(traba jo21, trabajo22) -diff (beneficios2 (trabajo21, trabajo 22), trabajo22):

solucion empresa:=solve ( d 11 (trabajo11, trabajo12)=0, d12 ( trabajo11, trabajo12) =0, d21 (trabajo21, trabajo22) =0, d22 (tr abajo21, trabajo22) $=0\},\{$ trabajo11, trabajo12, trabajo21, tra bajo22\}) : assign (solu cion_empresa);

demtrabajo11:=unapply (trabajo11, salario1) :demtrabajo12:= unapply (trabajo12, salario1,precio1, exr1) : demtrabajo21:=u napply (trabajo21, salario2) : demtrabajo22:=unapply (trabajo 
22, salario2, precio2, exr2):

demtrabajo1:=(salario1, precio1, exr1)-

demtrabajo11 (salario1)

+demtrabajo12 (salario1, precio1, exr1) :demtrabajo2:=(salar

io2, precio2, exr2) -

demtrabajo21 (salario2) +demtrabajo22 (salario2,

precio2, exr2):

ofbienes1:=salario1-

produccion1 (demtrabajo11 (salario1)) : of

bienes $2:=$ salario2-produccion2 (demtrabajo21 (salario2)) :

ofexport $1:=($ salario1, precio1)-exportacion1 (demtrabajo12

(salario1, precio1, exr1)) : ofexport $2:=($ salario2,precio2) -

expor tacion2(demtrabajo22 (salario2, precio2, exr2)) :\#

oferta de exp.

ganancias1:=(precio1, salario1, exr1)-

preciol*ofbienes1 (salariol

) +exr1*ofexport1 (salario1, precio1, exr1)-

precio1*salariol*demtra

bajo1 (salario1, precio1, exr1) : ganancias2:=(precio2, salari

$02, \operatorname{exr} 2)-$

precio2*ofbienes 2 (salario2) +exr2*ofexport2 (salario2, prec

io2, exr2) -

precio2*salario2*demtrabajo2 (salario2, precio2, exr2):

trabajo1:='trabajo1' :trabajo2:='trabajo2' :dinero:='diner o':ofdinero='ofdinero':

excessbienes1:=(precio1, precio2, salario1, salario2, exr1, e $\operatorname{xr} 2)$ -

dembienes1 (precio1, precio2, salario1, salario2, ganancias1 ( precio1, salario1, exr1), ganancias2 (precio2, salario2, exr2) , exr1, exr2) tofdinero/precio1-ofbienes1 (salario1):

excessbienes $2:=($ precio1, precio2, salario1, salario2, exr1, e xr2) -

dembienes2 (precio1, precio2, salario1, salario2, ganancias1 ( precio1, salario1, exr1), ganancias2 (precio2, salario2, exr2) , exr1, exr2)-ofbienes2 (salario2)-ofdinero/precio2:

excesstrabajo1:=(precio1, precio2, salario1, salario2, exr1, exr2)-demtrabajo1 (salario1, precio1, exr1)-

oftrabajo1 (precio1, precio2,

salario1, salario2, ganancias1 (precio1, salario1, exr1), gana ncias2 (precio2, salario2, exr2), exr1, exr2) :

excesstrabajo2:=(precio1, precio2, salariol, salario2, exr1, exr2) -demtrabajo2 (salario2, precio2, exr2)-

oftrabajo2 (precio1, precio2,

salario1, salario2, ganancias1 (precio1, salario1, exr1), gana ncias2 (precio2, salario2, exr2), exr1, exr2) :

balanza:=(precio1, precio2, salario1, salario2, exr1, exr2)exr1

* demimport1 (precio1, precio2, salario1, salario2, ganancias1 (precio1, salario1, exr1), ganancias2 (precio2, salario2, exr2 ), exr1, exr2) +exr2* demimport2 (precio1, precio2, salario1, sa lario2, ganancias1 (precio1, salario1, exr1), ganancias2 (prec io2, salario2, exr2), exr1, exr2)-

exr1*ofexport1 (salario1, precio1, exr1) -

exr2*ofexport2 (sala rio2, precio2, exr2) :

precio1:=1:exr1:=precio1:salario1:='salariol':salario2:=

'salario2' :precio2:='precio2' : exr2:='precio2' :

alpha:=parámetro1: beta:= parámetro2: rho:= parámetro3: theta:= parámetro4: ofdinero:=parámetro5:\# Se incorporan 
valores.

equilibrio:=solve ( excesstrabajo1 (precio1, precio2, salari o1, salario2, exr1, exr2) =0, excesstrabajo2 (precio1, precio2, salario1, salario2, exr1, exr2) =0, excessbienes2 (precio1, pre cio2, salario1, salario2, exr1, exr2) =0, balanza (precio1, prec io2, salario1, salario2, exr1,

exr2) =0, precio20, salario10, salario20\}, \{precio2, salario1, salario2\}); assign(equilibrio):

depreciacion:=(precio2-precio1)/precio1;

cuentacorrientel:=ofexport1 (salario1, preciol, exr1) -

(demimport1 (precio1, precio2, salario1, salario2, ganancias1 (precio1, salario1, exr1), ganancias2 (precio2, salario2, exr2 ), $\operatorname{exr} 1, \operatorname{exr} 2))$;

cuentacorriente 2 :=ofexport2 (salario2, precio2, exr2) (demimport2 (precio1, precio2, salario1, salario2, ganancias1 (precio1, salario1, exr1), ganancias2 (precio2, salario2, exr2 )$, \operatorname{exr} 1, \operatorname{exr} 2))$;

nocomerciables1:=dembienes1 (precio1,precio2, salario1, sal ario2, ganancias1 (precio1, salario1, exr1), ganancias2 (preci o2, salario2, exr2), exr1, exr2);

PIB1:=ofbienes1 (salario1); X1:=ofexport1 (salario1, precio1 , exr1);

IM1:=demimport1 (precio1, precio2, salario1, salario2, gananc ias1 (precio1, salario1, exr1), ganancias2 (precio2, salario2, $\operatorname{exr} 2), \operatorname{exr} 1, \operatorname{exr} 2)$;

(36) 


\section{Referencias}

1. Aspe, P. (1993). "Economic Transformation: The Mexican Way", MIT Press, Cambridge, MA.

2. Adams C. y Gros D. 1986 "The consequences of real exchange rate rules for inflation: Some illustrative examples", IMF Staff Papers 33.

3. Ball L., Mankiw N.G., y Romer D. (1988) "The new Keynesian Economics and the OutputInflation Trade-off", Brookings Papers on Economic Activity, 1.

4. Bacha, E. (1979) "Notes on the Brazilian experience with mini-devaluations (1968-1976)", J ournal of Development Economics, 6.

5. Barro R. (1978) "Unanticipated Money, Output, and the Price Level in the United States", J ournal of Political Economy 86.

6. Blanchard O. (1986) "The Wage-Price Spiral", Quarterly Journal of Economics, 101.

7. Blanchard, O. y Summers, L. (1986) "Hysteresis and the European Unemployment Problem" NBER Macroeconomics Annual 1.

8. Blejer M. I. y Fernández R. B. (1980) "The Effects of Unanticipated Money Growth on Prices and on Output and its Composition in a Fixed-Exchange-Rate Open Economy", Canadian J ournal of Economics, XIII, February.

9. Calvo G.A., Reinhart C.M. y Vegh C.A. 1995 "Targeting the real exchange rate: Theory and evidence", Journal of Development Economics, Vol. 47.

10. Carstens, A. y F. Gil Díaz (1996). "Some hypothesis related to the Mexican 1994-95 crisis", Documentos de Investigación del Banco de México, 9601.

11. Caplin A. y Spulber D. (1987) "Menu Costs and the Neutrality of Money", Quarterly Journal of Economics, 102, 4, Nov.

12. Caplin A. y Leahy J. (1991) "State Dependent Pricing and the Dynamics of Money and Output", Quarterly J ournal of Economics, 106, 3, August.

13. Dornbusch, R. (1982) "PPP exchange rules and macroeconomic stability", Journal of Political Economy, 90.

14. Fischer (1977); Friedman, M. (1968) "The Role of Monetary Policy", American Economic Review 58 (March).

15. Gaytán, A. y E. Rocha (1996) "Pruebas de Cointegración sobre el Agregado Monetario M4 en México", mimeo, Banco de México.

16. Gordon R. J. (1996). "Comments on: The Macroeconomics of Low Inflation" by Akerlof G.A., Dickens W.T., and Perry G.L., Brookings Papers on Economic Activity.

17. J ournal of Economic Perspectives, Vol 11. Winter.

18. Harris, R. (1995) "Using Co-integration Analysis in Econometric Modeling", Prentice Hall, Hertfordshire. Reino Unido.

19. Hume, D. (1970) "Writings on Economics", editado por Eugene Rotwein, Madison, University of Winsconsin Press.

20. Kamin, S. (1996) "Real Exchange rates and Inflation in exchange-Rate based Stabilizations: an empirical examination", Board of Governors of the Federal Reserve System, International Finance Discussion Papers, 554 
21. Lucas R.E. Jr. (1972) "Expectations and the Neutrality of Money", Journal of Economic Theory, vol 4. April.

22. (1981) "Tobin and Monetarism: A Review Article" , Journal of Economic Literature, J une

23. Nobel Lecture: Monetary Neutrality" " Journal of Political Economy, vol. 104 , no. 104.

24. Lizondo J. S. 1993 "Real exchange rate targeting under imperfect asset substitutability, IMF Staff Papers 40.

25. Mc. Donald, R. (1997) "What Determines Real Exchange Rates? The Long and Short of It, I MF Working Paper, WP/97/21

26. Montiel P.J. y Ostry J. 1991 "Macroeconomic implications of real exchange rate targeting in developing countries", IMF Staff Papers 38.

27. 1992 "Real exchange rate targeting under capital controls: Can money provide a nominal anchor?", IMF Staff Papers 39.

28. Phelps, E.S. (1968) "Money-Wage Dynamics and Labor Market Equilibrium", Journal of Political Economy 76 ( July/August).

29. Phillips A.W. (1958) "The Relationship between Unemployment and the Rate of Change of Money Wages in the United Kingdom, 1861-1957", Economica 25 (November).

30. Reynoso, A. (1989) "Essays on the Macroeconomic Effects of Monetary Reforms, Price Controls and Financial Repression" Tesis Doctoral, MIT.

31. (1997) "Macroeconomía Interactiva: Un curso de Macroeconomía Intermedia con MapleV", mimeo, ITAM.

32. Romer D. (1996) Advanced Macroeconomics, Mc Graw-Hill, 1996.

33. Samuelson, P.A. (1958) "An exact Consumption-loan Model of Interest with or without the Social Contrivance of Money", J ournal of Political Economy, 66. December.

34. Stiglitz J. (1997) "Reflections on the Natural Rate Hypothesis", Journal of Economic Perspectives, Vol. 11. Winter.

35. Staiger D., Stock J.H., and Watson M.W. (1997) "The NAIRU, Unemployment and Monetary Policy", J ournal of Economic Perspectives, Vol 11. Winter.

36. Taylor J.B. (1979) "Estimation and Control of a Macroeconomic Model with Rational Expectations", Econometrica, 47, September.

37. Maintaining Long-Run Price Stability -.- Conceptual Issues", in Achieving Price Stability, Symposium Sponsored by the Federal Reserve Bank of Kansas City.

38. Tinbergen, Jan (1952) "On the Theory of Economic Policy", 2\&ordf;. Edición, Amsterdam, North Holland. 